\title{
Estimating censored regression models in the presence of nonparametric multiplicative heteroskedasticity
}

\author{
Songnian Chen ${ }^{\mathrm{a}}$, Shakeeb Khan ${ }^{\mathrm{b}, *}$ \\ ${ }^{\mathrm{a}}$ Finance and Economics School of Business and Management, The Hong Kong University of Science \\ and Technology, Clear Water Bay, Kowloon, Hong Kong \\ ${ }^{\mathrm{b}}$ Department of Economics, University of Rochester, Rochester, NY 14627, USA
}

Received 15 October 1998; received in revised form 6 January 2000; accepted 13 March 2000

\begin{abstract}
Powell's (1984, Journal of Econometrics 25, 303-325) censored least absolute deviations (CLAD) estimator for the censored linear regression model has been regarded as a desirable alternative to maximum likelihood estimation methods due to its robustness to conditional heteroskedasticity and distributional misspecification of the error term. However, the CLAD estimation procedure has failed in certain empirical applications due to the restrictive nature of the 'full rank' condition it requires. This condition can be especially problematic when the data are heavily censored. In this paper we introduce estimation procedures for heteroskedastic censored linear regression models with a much weaker identification restriction than that required for the LCAD, and which are flexible enough to allow for various degrees of censoring. The new estimators are shown to have desirable asymptotic properties and perform well in small-scale simulation studies, and can thus be considered as viable alternatives for estimating censored regression models, especially for applications in which the CLAD fails. (C) 2000 Elsevier Science S.A. All rights reserved.
\end{abstract}

JEL classification: C14; C23; C24

Keywords: Censored regression; Full rank condition; Heavy censoring; Multiplicative heteroskedasticity

\footnotetext{
* Corresponding author.

E-mail address: skhan@troi.cc.rochester.edu (S. Khan).
} 


\section{Introduction and motivation}

The censored regression model, sometimes referred to by economists as the 'Tobit' model, has been the focus of much attention in both the applied and theoretical econometrics literature since the seminal work of Tobin (1958). In its simplest form the model is written as

$$
y_{i}=\max \left(x_{i}^{\prime} \beta_{0}+\varepsilon_{i}, 0\right),
$$

where $y_{i}$ is an observable response variable, $x_{i}$ is a $d$-dimensional vector of observable covariates, $\varepsilon_{i}$ is an unobservable error term, and $\beta_{0}$ is the $d$ dimensional 'parameter of interest'. As many economic data sets are subject to various forms of censoring, the development of consistent estimation procedures for this model and variations thereof has become increasingly important. Traditionally this model has been estimated by maximum likelihood methods after imposing homoskedasticity and parametric restrictions on the underlying error terms. More recently a number of consistent estimators have been proposed which allow for much weaker restrictions on the error terms, such as constant conditional quantiles (Powell, 1984, 1986a; Nawata, 1990; Khan and Powell, 1999; Buchinsky and Hahn, 1998), conditional symmetry (Powell, 1986b) and independence between the errors and regressors (Horowitz, 1986, 1988; Moon, 1989; Honore and Powell, 1994). The weakest such restriction is the constant conditional quantile restriction. Powell (1984) exploited a conditional median restriction on the error term, and proposed the censored least absolute deviations (CLAD) estimator, defined as the minimizer of

$$
S_{n}(\beta)=\frac{1}{n} \sum_{i=1}^{n}\left|y_{i}-\max \left(x_{i}^{\prime} \beta, 0\right)\right| .
$$

This median restriction was generalized to any specific quantile, $\alpha \in(0,1)$ in Powell (1986a), generalizing the objective function to

$$
Q_{n}(\beta)=\frac{1}{n} \sum_{i=1}^{n} \rho_{\alpha}\left(y_{i}-\max \left(0, x_{i}^{\prime} \beta\right)\right),
$$

where $\rho_{\alpha}(\cdot) \equiv \alpha|\cdot|+(2 \alpha-1)(\cdot) I[\cdot<0]$ (where $I[\cdot]$ is the indicator function) denotes the 'check' function introduced in Koenker and Bassett (1978). These estimators are very attractive due to weak assumptions they require, making them 'robust' to conditional heteroskedasticity and non-normality of the error distribution. Furthermore, Powell (1984, 1986a) showed that these estimators have desirable asymptotic properties, notably their parametric $(\sqrt{n})$ rate of convergence, and limiting normal distribution.

However, there is one serious drawback to the use of the CLAD estimator which has been encountered in certain practical applications. The CLAD 
estimator requires the matrix

$$
E\left[I\left[x_{i}^{\prime} \beta_{0}>0\right] x_{i} x_{i}^{\prime}\right]
$$

to be of full rank $^{1}$ for identification of $\beta_{0}$. In the empirical work of Chay (1995) and Honore et al. (1997) the CLAD estimator ${ }^{2}$ has failed in the sense that the sample analog of the full rank condition could not be satisfied for estimates of $\beta_{0}$ which minimized (1.2). In general, this full rank condition creates stability problems for the CLAD estimation procedure in data sets where the index $x_{i}^{\prime} \beta_{0}$ is negative with a high probability, as would usually be the case when the data are heavily censored. In empirical settings, heavily censored data are frequently encountered in household expenditure data, where a significant number of zero expenditures are observed. Examples include Jarque (1987) and Melenberg and Van Soest (1996). The drawbacks of median-based estimation in the analysis of this type of data serve as one of the motivations for the approach based on second moments proposed by Lewbel and Linton (1998).

This full rank condition becomes more 'flexible' if we minimize (1.3) instead of (1.2) using a quantile $\alpha>0.5$. In this case we can write the full rank condition as

$$
E\left[I\left[q_{\alpha}\left(x_{i}\right)>0\right] x_{i} x_{i}^{\prime}\right],
$$

where $q_{\alpha}\left(x_{i}\right)$ denotes the $\alpha$ th conditional quantile function. Since $q_{\alpha}\left(x_{i}\right) \geqslant$ $q_{0.5}\left(x_{i}\right)$ when $\alpha>0.5$ this condition is more likely to be satisfied in practical applications.

However, estimating $\beta_{0}$ through the minimization of (1.3) using various quantiles necessarily rules out the possibility of conditional heteroskedasticity, since it requires that all conditional quantiles are constant. For any statistical model in which conditional heteroskedasticity is present, the most sensible location restriction to impose on the error terms is a conditional mean or median restriction. It is well known that the censored regression model is not identified under a conditional mean restriction, leaving the conditional median restriction, and hence the identification restriction in (1.4), as necessary for estimating $\beta_{0}{ }^{3}$ In other words, when $\beta_{0}$ is the fixed parameter of interest (interpreted for example as the change in the conditional median of the response

\footnotetext{
${ }^{1}$ It should be noted that this full rank condition is necessary given the conditional quantile restriction (see Powell, 1984, 1986a). Thus it is also needed for estimators in the literature based on this restriction, such as Nawata (1990), Khan and Powell (1999), Buchinsky and Hahn (1998).

${ }^{2}$ In the work of Honoré et al. (1997), the actual CLAD estimator was not used in estimation. An alternative procedure, based on an analogous identification condition, was proposed and failed in its empirical application.

${ }^{3}$ This point is also mentioned in Powell (1986a), where he acknowledges that in the presence of heteroskedasticity, setting the median of the error term to 0 is crucial to the interpretation of $\beta_{0}$ as the coefficients of the 'typical response' of the censored dependent variable to the regressors.
} 
variable given a one unit change in the regressors), if conditional heteroskedasticity is to be allowed for, $\alpha$ must be fixed a priori, and not determined by the degree of censoring in the data set.

In this paper we aim to address this problem by proposing estimators for the censored regression model which permit conditional heteroskedasticity, yet allow for much less stringent identification conditions than that required by the CLAD. We do so by restricting the structural form of conditional heteroskedasticity to be multiplicative, modelling the error term as the product of a nonparametrically specified 'scale' function of the regressors, and a homoskedastic error term:

$$
\varepsilon_{i}=\sigma\left(x_{i}\right) u_{i} \quad \mathrm{P}\left(u_{i} \leqslant \lambda \mid x_{i}\right) \equiv \mathrm{P}\left(u_{i} \leqslant \lambda\right) \forall \lambda \in \mathbb{R}, x_{i} \text { a.s. }
$$

Note that this structure still allows for conditional heteroskedasticity of very general forms, as $\sigma(\cdot)$ is left unspecified.

Multiplicative heteroskedasticity has been adopted in various forms for many models in the econometric and statistics literature. There are many examples of such structures where the scale function is parametrically specified. For example, in the time series literature, the ARCH model introduced by Engle (1982) assumes this type of structure. In modelling cross-sectional data, Judge et al. (1982) explain why multiplicative heteroskedasticity may be present when modelling the expenditure/income relationship, or estimating average cost curves. Other relevant examples are Harvey (1976) and Melenberg and Van Soest (1996). Furthermore, in the context of limited dependent variable models, many of the tests for heteroskedasticity consider alternatives which have a multiplicative structure; examples include Koenker and Bassett (1982), Powell (1986a) and Maddala (1995). In the estimation of conditional distribution index models, Newey and Stoker (1993) consider a class of models subject to (nonparametric) multiplicative heteroskedasticity. Finally, in nonparametric estimation, multiplicative structures to model heteroskedasticity is considered in Fan and Gijbels (1996), and Lewbel and Linton (1998). ${ }^{4}$

We propose two estimation procedures, based on two restrictions on the homoskedastic error term $u_{i}$. The first estimator is based on the assumption that $u_{i}$ has a known distribution, ${ }^{5}$ and without loss of generality we assume that it is known to have a standard normal distribution. While this restricts the error term behavior a great deal further, it may not be that serious of an assumption. For example, it has been concluded in Powell (1986a), Donald (1995), and

\footnotetext{
${ }^{4}$ Lewbel and Linton (1998) estimate censored regression models. Their approach is different from what is done in this paper in that their latent equation is nonparametrically specified, and they propose an estimator of the unknown location function. Here, we consider a semiparametric model, and propose an estimator for the finite-dimensional parameters.

${ }^{5}$ More precisely, we assume that the quantiles of $u_{i}$ are known to the econometrician.
} 
Horowitz (1993) that heteroskedasticity is a far more serious problem than departures from normality when estimation of $\beta_{0}$ is concerned. Their conclusions are consistent with our simulation results discussed later in this paper. The second estimation procedure we introduce allows us to do away with the known distribution assumption, only requiring that $u_{i}$ have a positive density function on the real line.

As detailed in the next section, both estimators involve two stages. The first stage involves nonparametric quantile regression, and the second stage adopts a simple least-squares type fitting device.

The paper is organized as follows. The following section motivates the estimation procedures we propose and discusses each of the two stages involved in greater detail. Section 3 outlines regularity conditions used in proving the asymptotic properties of the estimators, and then outlines the steps involved for the proof. Section 4 explores the finite sample properties of these estimators through a small-scale simulation study, and compares their performance to the CLAD estimator. Finally, Section 5 concludes by summarizing our results and examining possible extensions and areas for future research.

\section{Models and estimation procedures}

We consider censored regression models of the form

$$
\begin{aligned}
y_{i} & =\max \left(x_{i}^{\prime} \beta_{0}+\varepsilon_{i}, 0\right), \\
\varepsilon_{i} & =\sigma\left(x_{i}\right) u_{i} .
\end{aligned}
$$

The only restriction we impose on the 'scale' function $\sigma(\cdot)$ is that it be positive and satisfy certain 'smoothness' properties, as detailed in the next section. We first consider an estimator based on a normality assumption on $u_{i}$ :

$$
\mathrm{P}\left(u_{i} \leqslant \lambda \mid x_{i}\right)=\Phi(\lambda)
$$

where $\Phi(\cdot)$ denotes the c.d.f. of the standard normal distribution. ${ }^{6}$ To construct an estimator for $\beta_{0}$ based on this restriction, we note that for any quantile $\alpha \in(0,1)$, an equivariance property of quantiles implies that

$$
q_{\alpha}\left(x_{i}\right)=\max \left(x_{i}^{\prime} \beta_{0}+c_{\alpha}^{Z} \sigma\left(x_{i}\right), 0\right),
$$

where $q_{\alpha}(\cdot)$ denotes the $\alpha$ th conditional quantile function and $c_{\alpha}^{Z}$ denotes the $\alpha$ th quantile of the standard normal distribution.

\footnotetext{
${ }^{6}$ Note that setting the variance of $u_{i}$ to one is just a normalization that is required by leaving $\sigma(\cdot)$ unspecified.
} 
Thus if $q_{\alpha_{i}}\left(x_{i}\right)>0$ for two distinct quantiles $\alpha_{1}$ and $\alpha_{2}$, and some point $x_{i}$, then we can combine the relations

$$
\begin{aligned}
& q_{\alpha_{1}}\left(x_{i}\right)=x_{i}^{\prime} \beta_{0}+c_{\alpha_{1}}^{Z} \sigma\left(x_{i}\right), \\
& q_{\alpha_{2}}\left(x_{i}\right)=x_{i}^{\prime} \beta_{0}+c_{\alpha_{2}}^{Z} \sigma\left(x_{i}\right)
\end{aligned}
$$

to yield the relationship ${ }^{7}$

$$
\frac{c_{\alpha_{2}}^{Z} q_{\alpha_{1}}\left(x_{i}\right)-c_{\alpha_{1}}^{Z} q_{\alpha_{2}}\left(x_{i}\right)}{c_{\alpha_{2}}^{Z}-c_{\alpha_{1}}^{Z}}=x_{i}^{\prime} \beta_{0} .
$$

This suggests that if the values of the quantile functions were known for $d$ observations, the parameter of interest $\beta_{0}$ could be recovered by solving the above system of equations. The condition necessary for a unique solution to this system is the full rank of the matrix

$$
I\left[q_{\alpha_{1}}\left(x_{i}\right)>0\right] x_{i} x_{i}^{\prime}
$$

assuming, without loss of generality, that $\alpha_{1}<\alpha_{2}$. This generalizes the full rank condition of the CLAD estimator as long as $\alpha_{1}>0.5$. Of course, the above system of equations cannot immediately translate into an estimator of $\beta_{0}$ since the values of the conditional quantile functions $q_{\alpha}(\cdot)$ are unknown. However, they can be estimated nonparametrically in a preliminary stage, and these estimated values $\left(\hat{q}_{\alpha}(\cdot)\right)$ can be 'plugged' into the system of equations, defining a two-step estimator of $\beta_{0}$ as

$$
\widehat{\beta}=\left[\frac{1}{n} \sum_{i=1}^{n} I\left[\hat{q}_{\alpha_{1}}\left(x_{i}\right)>0\right] x_{i} x_{i}^{\prime}\right]^{-1} \frac{1}{n} \sum_{i=1}^{n} \hat{y}_{i} x_{i}
$$

where

$$
\hat{y}_{i} \equiv \frac{c_{\alpha_{2}}^{Z} \hat{q}_{\alpha_{1}}\left(x_{i}\right)-c_{\alpha_{1}}^{Z} \hat{q}_{\alpha_{2}}\left(x_{i}\right)}{c_{\alpha_{2}}^{Z}-c_{\alpha_{1}}^{Z}} .
$$

We next consider relaxing the normality assumption on $u_{i}$, only requiring that it have a continuous distribution with a positive density function. In contrast to the previous estimator, which treats the two quantile function values as pseudodependent variables, we will now treat the average of the quantile functions as a pseudo-dependent variable and their difference as a pseudo-regressor. Specifically, letting $c_{\alpha_{i}}$ denote the (unknown) quantile values of $u_{i}$, for a value of

\footnotetext{
${ }^{7}$ This relationship illustrates that $u_{i}$ need not have a normal distribution for the estimator to be applicable. All that is required is that the distribution of $u_{i}$ be known, so the appropriate quantiles $c_{\alpha_{1}}, c_{\alpha_{2}}$ are used.
} 
$x_{i}$ where both quantile functions are positive, we have the following relationship:

$$
\bar{q}\left(x_{i}\right)=x_{i}^{\prime} \beta_{0}+\frac{\bar{c}}{\Delta c} \Delta q\left(x_{i}\right)
$$

where $\quad \bar{q}(\cdot) \equiv\left(q_{\alpha_{2}}(\cdot)+q_{\alpha_{1}}(\cdot)\right) / 2, \Delta q(\cdot) \equiv q_{\alpha_{2}}(\cdot)-q_{\alpha_{1}}(\cdot), \bar{c} \equiv\left(c_{\alpha_{2}}+c_{\alpha_{1}}\right) / 2$, $\Delta c \equiv c_{\alpha_{2}}-c_{\alpha_{1}}$. This suggests an alternative second stage least-squares fitting device, regressing $\hat{q}(\cdot)$ on $x_{i}$ and $\Delta \hat{q}(\cdot)$. Specifically, let $\hat{\beta} \in \mathbb{R}^{d}$ and the 'nuisance parameter' $\hat{\gamma}_{1} \in \mathbb{R}$ minimize the least-squares function

$$
\frac{1}{n} \sum_{i=1}^{n} I\left[\hat{q}_{\alpha_{1}}\left(x_{i}\right)>0\right]\left(\hat{q}\left(x_{i}\right)-x_{i}^{\prime} \beta-\gamma_{1} \Delta \hat{q}\left(x_{i}\right)\right)^{2} .
$$

We note that both estimators are defined as functions of nonparametric estimators, which are known to converge at a rate slower than $\sqrt{n}$. The next section shows how the second stage estimators can still achieve the parametric rate of convergence under regularity conditions which are common in the literature.

Before proceeding to a discussion of the asymptotic properties of these estimators we first discuss each of the stages of our two procedures in greater detail. Specifically, we discuss the nonparametric estimation procedure adopted in the first stage, and some technical complications which require the modification of the second stage objective functions.

\subsection{First stage of the estimators}

The first stage involves nonparametrically estimating the conditional $\alpha_{1}$, $\alpha_{2}$ quantiles of the observed dependent variable $y_{i}$ given the regressors $x_{i}$. While several conditional quantile estimators have been recently proposed in the statistics and econometrics literature, we use the local polynomial estimator introduced in Chaudhuri (1991a,b). This estimation procedure is computationally simple (it involves minimization of a globally convex objective function which can be handled using linear programming methods) and it allows for simple control of the order of the bias by selecting the appropriate order of the polynomial. Its description is facilitated by introducing new notation, and the notation adopted has been chosen deliberately to be as close as possible to that introduced in Chaudhuri (1991a,b).

First, we assume that the regressor vector $x_{i}$ can be partitioned as $\left(x_{i}^{(\mathrm{ds})}, x_{i}^{(\mathrm{c})}\right)$, where the $d_{\mathrm{ds}}$-dimensional vector $x_{i}^{(\mathrm{ds})}$ is discretely distributed, and the $d_{\mathrm{c}}$ dimensional vector $x_{i}^{(\mathrm{c})}$ is continuously distributed.

We let $C_{n}\left(x_{i}\right)$ denote the cell of observation $x_{i}$ and let $h_{n}$ denote the sequence of bandwidths which govern the size of the cell. For some observation $x_{j}, j \neq i$, we let $x_{j} \in C_{n}\left(x_{i}\right)$ denote that $x_{j}^{(\mathrm{ds})}=x_{i}^{(\mathrm{ds})}$ and $x_{j}^{(\mathrm{c})}$ lies in the $d_{\mathrm{c}}$-dimensional cube centered at $x_{i}^{(\mathrm{c})}$ with side length $2 h_{n}$. 
Next, we let $k$ denote the assumed order of differentiability of the quantile functions with respect to $x_{i}^{(\mathrm{c})}$ and we let $A$ denote the set of all $d_{\mathrm{c}}$-dimensional vectors of nonnegative integers $b_{l}$, where the sum of the components of each $b_{l}$, which we denote by $\left[b_{l}\right]$, is less than or equal to $k$. We order the elements of the set $A$ such that its first element corresponds to $\left[b_{l}\right]=0$, and we let $s(A)$ denote the number of elements in $A$. A simple example will help illustrate this notation. Suppose the quantile function is assumed to be twice differentiable, and $x_{i}$ consists of two continuous components. Then $A$ would correspond to the set of vectors $(0,0),(1,0),(0,1),(1,1),(2,0),(0,2)$, so $s(A)=6$.

For any $s(A)$-dimensional vector $\theta$, we let $\theta_{(l)}$ denote its $l$ th component, and for any two $s(A)$-dimensional vectors $a, b$, we let $a^{b}$ denote the product of each component of $a$ raised to the corresponding component of $b$. Finally, we let $I[\cdot]$ be an indicator function, taking the value 1 if its argument is true, and 0 otherwise. The local polynomial estimator of the conditional $\alpha$ th quantile function at a point $x_{i}$ for any $\alpha \in(0,1)$ involves $\alpha$-quantile regression (see Koenker and Bassett, 1978) on observations which lie in the defined cells of $x_{i}$. Specifically, let the vectors

$$
\left(\hat{\theta}_{(1)}, \hat{\theta}_{(2)}, \ldots, \hat{\theta}_{(s(A))}\right)
$$

minimize the objective function ${ }^{8}$

$$
\sum_{j=1}^{n} I\left[x_{j} \in C_{n}\left(x_{i}\right)\right] \rho_{\alpha}\left(y_{i}-\sum_{l=1}^{s(A)} \theta_{(l)}\left(x_{j}^{(\mathrm{c})}-x_{i}^{(\mathrm{c})}\right)^{b_{l}}\right),
$$

where we recall that $\rho_{\alpha}(x) \equiv \alpha|x|+(2 \alpha-1) x I[x<0]$. The conditional quantile estimator which will be used in the first stage will be the value $\hat{\theta}_{(1)}$ corresponding to the two selected values of $\alpha$.

The motivation for including a higher-order polynomial in the objective function and estimating the nuisance parameters $\left(\hat{\theta}_{(2)}, \ldots, \hat{\theta}_{(s(A))}\right)$ is to achieve bias reduction of the nonparametric estimator, analogous to using a 'higherorder' kernel with kernel estimation. This will be necessary to achieve the parametric rate of convergence for the second stage estimator.

\subsection{Second stage of each estimator}

The second stage of our estimators treat the values estimated in the first stage as 'raw' data, and adopts weighted least-squares type fitting devices to estimate $\beta_{0}$. As mentioned previously, positive weight will only be given to observations whose estimated quantile function values exceed the censoring point. We thus

\footnotetext{
${ }^{8}$ For technical reasons used in proving asymptotic properties of the second stage estimator, we actually require that this objective function be minimized over a compact subset of $\mathbb{R}^{s(A)}$.
} 
propose minimizing second stage objective functions of the form

$$
\frac{1}{n} \sum_{i=1}^{n} \tau\left(x_{i}\right) \omega\left(\hat{q}_{\alpha_{1}}\left(x_{i}\right)\right)\left(\hat{y}_{i}-x_{i}^{\prime} \beta\right)^{2},
$$

where $\hat{y}_{i}=\left(c_{\alpha_{1}}^{Z} \hat{q}_{\alpha_{2}}\left(x_{i}\right)-c_{\alpha_{1}}^{Z} \hat{q}_{\alpha_{1}}\left(x_{i}\right)\right) /\left(c_{\alpha_{2}}^{Z}-c_{\alpha_{1}}^{Z}\right)$, for the estimator under the normality assumption, and

$$
\frac{1}{n} \sum_{i=1}^{n} \tau\left(x_{i}\right) \omega\left(\hat{q}_{\alpha_{1}}\left(x_{i}\right)\right)\left(\hat{q}\left(x_{i}\right)-x_{i}^{\prime} \beta-\Delta \hat{q}\left(x_{i}\right) \gamma_{1}\right)^{2}
$$

without the normality assumption. Here $\omega(\cdot)$ is a 'smooth' weighting function which only keeps observations for which the first stage estimation values exceed the censoring value, and $\tau(\cdot)$ is a trimming function, whose support is a compact subset of $\mathbb{R}^{d}$, which we denote by $\mathscr{X}$. This yields closed-form, least-squares type estimators of the form

$$
\widehat{\beta}=\left(\frac{1}{n} \sum_{i=1}^{n} \tau\left(x_{i}\right) \omega\left(\hat{q}_{\alpha_{1}}\left(x_{i}\right)\right) x_{i} x_{i}^{\prime}\right)^{-1} \frac{1}{n} \sum_{i=1}^{n} \tau\left(x_{i}\right) \omega\left(\hat{q}_{\alpha_{1}}\left(x_{i}\right)\right) x_{i} \hat{y}_{i}
$$

and, letting $\hat{\gamma}$ denote our second estimator for $\gamma_{0} \equiv\left(\beta_{0}^{\prime}, \gamma_{1}\right)^{\prime}$,

$$
\hat{\gamma}=\left(\frac{1}{n} \sum_{i=1}^{n} \tau\left(x_{i}\right) \omega\left(\hat{q}_{\alpha_{1}}\left(x_{i}\right)\right) \hat{z}_{i} \hat{z}_{i}^{\prime}\right)^{-1} \frac{1}{n} \sum_{i=1}^{n} \tau\left(x_{i}\right) \omega\left(\hat{q}_{\alpha_{1}}\left(x_{i}\right)\right) \hat{z}_{i} \hat{\bar{q}}\left(x_{i}\right),
$$

where $\hat{z}_{i}$ denotes the vector $\left(x_{i}^{\prime}, \Delta \hat{q}\left(x_{i}\right)\right)^{\prime}$. We note that the proposed estimators fall into the class of 'semiparametric two-step' estimators, for which many general asymptotic results have been developed (see Andrews, 1994; Newey and McFadden, 1994; Sherman, 1994). As established in the next section, under appropriate regularity conditions the parametric $(\sqrt{n})$ rate of convergence can be obtained for the second stage estimators despite the nonparametric rate of convergence of the first stage estimator.

\section{Asymptotic properties of the estimators}

The necessary regularity conditions will first be outlined in detail before proceeding with the consistency and asymptotic normality results. Specific assumptions are imposed on the distribution of the errors and regressors, the order of smoothness of the scale function $\sigma(\cdot)$, and the bandwidth sequence conditions needed for the first stage.

\subsection{Assumptions}

Assumption FR (Full rank conditions). Letting $J$ denote the $d \times d$ matrix

$$
E\left[\tau\left(x_{i}\right) \omega\left(q_{\alpha_{1}}\left(x_{i}\right)\right) x_{i} x_{i}^{\prime}\right]
$$


and letting $J^{*}$ denote the $(d+1) \times(d+1)$ matrix

$$
E\left[\tau\left(x_{i}\right) \omega\left(q_{\alpha_{1}}\left(x_{i}\right)\right) z_{i} z_{i}^{\prime}\right],
$$

where $z_{i} \equiv\left(x_{i}^{\prime}, \Delta q\left(x_{i}\right)\right)^{\prime}$, we assume that $J$ and $J^{*}$ are of full rank.

Assumption RS (Random sampling). The sequence of $d+1$ dimensional vectors $\left(\varepsilon_{i}, x_{i}\right)$ are independent and identically distributed.

Assumption WF (Weighting function properties). The weighting function, $\omega\left(\cdot: \mathbb{R} \rightarrow \mathbb{R}^{+}\right.$has the following properties:

WF.1. $\omega \equiv 0$ if its argument is less than $c$, an arbitrarily small positive constant.

WF.2. $\omega(\cdot)$ is differentiable with bounded derivative.

Assumption RD (Regressor distribution). We let $f_{X^{(\mathrm{c})} \mid X^{(\mathrm{d})}}\left(\cdot \mid x^{(\mathrm{ds})}\right)$ denote the conditional density function of $x_{i}^{(\mathrm{c})}$ given $x_{i}^{(\mathrm{ds})}=x^{(\mathrm{ds})}$, and assume it is bounded away from 0 and infinity on $\mathscr{X}$.

We let $f_{X^{(\mathrm{ds})}}(\cdot)$ denote the mass function of $x_{i}^{(\mathrm{ds})}$, and assume a finite number of mass points on $\mathscr{X}$.

Also, we let $f_{X}(\cdot)$ denote $f_{X^{(\mathrm{c})} \mid X^{(\mathrm{de})}}(\cdot \mid \cdot) f_{X^{(\mathrm{ds})}}(\cdot)$.

Assumption ED (Error density). The error terms $\varepsilon_{i}$ are of the form $\varepsilon_{i}=\sigma\left(x_{i}\right) u_{i}$ where $\sigma(\cdot)$ is a deterministic function of the regressors, and $u_{i}$ is a random variable distributed independent of the regressors. We assume $u_{i}$ has the following properties:

ED.1. If the first proposed estimator is used, $u_{i}$ is assumed to have a standard normal distribution.

ED.1'. If the second proposed estimator is used, it is only required that $u_{i}$ has a continuous distribution with density function that is bounded, positive, and continuous on $\mathbb{R}$.

Assumption OS (Orders of smoothness). For some $\varrho \in(0,1]$, and any real-valued

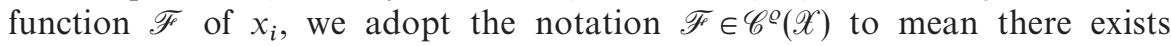
a positive constant $K<\infty$ such that

$$
\left|\mathscr{F}\left(x_{1}\right)-\mathscr{F}\left(x_{2}\right)\right| \leqslant K\left\|x_{1}-x_{2}\right\|^{\varrho}
$$

for all $x_{1}, x_{2} \in \mathscr{X}$. With this notation, we assume the following smoothness conditions:

OS.1. $f_{X}(\cdot), \tau(\cdot) \in \mathscr{C}^{\complement}(\mathscr{X})$.

OS.2. $\sigma(\cdot)$ is continuously differentiable in $x_{i}^{(\mathrm{c})}$ of order $k$, with $k$ th-order derivatives $\in \mathscr{C}^{\varrho}(\mathscr{X})$. We let $p=k+\varrho$ denote the order of smoothness of this function. 
Assumption BC (Bandwidth conditions). The first stage bandwidth sequence, denoted by $h_{n}$ is of the form

$$
h_{n}=c^{*} n^{-\xi},
$$

where $c^{*}$ is some positive constant, and $\xi \in\left(1 / 2 p, 1 / 3 d_{\mathrm{c}}\right)$, with $d_{\mathrm{c}}$ denoting the number of continuously distributed regressors.

\subsubsection{Remarks on the assumptions}

1. Assumption FR characterizes the 'full rank' conditions which illustrate the advantages of these estimators over Powell's CLAD estimator. The first such condition can be roughly interpreted as a full rank requirement on the matrix

$$
E\left[I\left[x_{i}^{\prime} \beta_{0}+c_{\alpha_{1}}^{Z} \sigma\left(x_{i}\right)>0\right] x_{i} x_{i}^{\prime}\right]
$$

which, by appropriate selection of the quantile $\alpha_{1}$, is less stringent than Powell's condition

$$
E\left[I\left[x_{i}^{\prime} \beta_{0}>0\right] x_{i} x_{i}^{\prime}\right] .
$$

2. The full rank condition imposed on $J^{*}$ will not be satisfied if $\varepsilon_{i}$ is homoskedastic. This problem can be overcome by first testing for heteroskedasticity in the data using either the test discussed in Powell (1986a) or a Hausmantype test comparing our estimator to an estimator based on an independence restriction, such as Honoré and Powell (1994). If heteroskedasticity is detected, one can use the approach mentioned here, as the full rank condition is satisfied. If heteroskedasticity is not detected by the tests, one can use Powell's approach with higher quantiles to estimate $\beta_{0}$ when the data are heavily censored, or use other estimators in the literature based on an independence restriction. This sequential procedure is analogous to the approach discussed in White (1980) for improving inference in the linear model. $^{9}$

3. Assumption WF imposes additional restrictions on the weighting function. It ensures that estimation is based only on observations for which the conditional quantile functions are greater than the censoring values. It is essentially a smooth approximation to an indicator function, that will help avoid certain technical difficulties. Note that Assumption WF.1 implies that the support of the weighting function is bounded away from the censoring value; this is necessary to avoid the 'boundary problem' that arises when nonparametric estimation procedures are used with censored data.

\footnotetext{
${ }^{9}$ It is also worth noting that the slope coefficients are still estimable in the homoskedastic case, though the intercept term is not. This can be shown by verifying the estimability condition on p. 58 of Amemiya (1985).
} 
4. Assumption $\mathrm{BC}$ allows for a range of bandwidth sequences, but rules out the sequence which yields the optimal rate of convergence of the first step estimator as discussed in Chaudhuri (1991b). It imposes 'undersmoothing' of the first stage nonparametric estimator.

\subsection{Limiting distribution of the estimators}

In this section it is established that under the assumptions detailed in the previous section, the proposed (second stage) estimators for the slope coefficients converge at the parametric $(\sqrt{n})$ rate, with asymptotic normal distributions. Before stating the main theorem, we let $v_{1 i}, v_{2 i}$ denote the 'residuals' $y_{i}-q_{\alpha_{1}}\left(x_{i}\right), y_{i}-q_{\alpha_{2}}\left(x_{i}\right)$ with conditional density functions denoted by $f_{v_{1} \mid X}$, $f_{v_{2} \mid X}$, and define the following mean 0 random vectors:

$$
\begin{aligned}
& \delta_{\alpha_{1}}\left(y_{i}, x_{i}\right)=\tau\left(x_{i}\right) \omega\left(q_{\alpha_{1}}\left(x_{i}\right)\right) f_{v_{1} \mid X}^{-1}\left(0 \mid x_{i}\right) \frac{c_{\alpha_{2}}^{Z}}{\Delta c^{Z}}\left(\alpha_{1}-I\left[y_{i} \leqslant q_{\alpha_{1}}\left(x_{i}\right)\right]\right) x_{i}, \\
& \delta_{\alpha_{2}}\left(y_{i}, x_{i}\right)=\tau\left(x_{i}\right) \omega\left(q_{\alpha_{1}}\left(x_{i}\right)\right) f_{v_{2} \mid X}^{-1}\left(0 \mid x_{i}\right) \frac{c_{\alpha_{1}}^{Z}}{\Delta c^{Z}}\left(\alpha_{2}-I\left[y_{i} \leqslant q_{\alpha_{2}}\left(x_{i}\right)\right]\right) x_{i}, \\
& \delta_{\alpha_{1}}^{*}\left(y_{i}, x_{i}, z_{i}\right)=\tau\left(x_{i}\right) \omega\left(q_{\alpha_{1}}\left(x_{i}\right)\right) f_{v_{1} \mid X}^{-1}\left(0 \mid x_{i}\right) \frac{c_{\alpha_{2}}}{\Delta c}\left(\alpha_{1}-I\left[y_{i} \leqslant q_{\alpha_{1}}\left(x_{i}\right)\right]\right) z_{i}, \\
& \delta_{\alpha_{2}}^{*}\left(y_{i}, x_{i}, z_{i}\right)=\tau\left(x_{i}\right) \omega\left(q_{\alpha_{1}}\left(x_{i}\right)\right) f_{v_{2} \mid X}^{-1}\left(0 \mid x_{i}\right) \frac{c_{\alpha_{1}}}{\Delta c}\left(\alpha_{2}-I\left[y_{i} \leqslant q_{\alpha_{2}}\left(x_{i}\right)\right]\right) z_{i} .
\end{aligned}
$$

The following theorem, whose proof is left to the appendix, establishes the parametric rate of convergence and also characterizes the limiting covariance matrices of each estimator. The expressions for these matrices reflect the fact that the limiting variance depends primarily on the variance of the first stage estimator, as the expressions for $\delta_{\alpha_{t}}(\cdot, \cdot)$ and $\delta_{\alpha_{t}}^{*}(\cdot, \cdot, \cdot), t=1,2$, are very similar to the influence function in the local Bahadur representation derived in Chaudhuri (1991a).

Theorem 1. Let $\Omega$ denote the $d \times d$ matrix

$$
E\left[\left(\delta_{\alpha_{2}}\left(y_{i}, x_{i}\right)-\delta_{\alpha_{1}}\left(y_{i}, x_{i}\right)\right)\left(\delta_{\alpha_{2}}\left(y_{i}, x_{i}\right)-\delta_{\alpha_{1}}\left(y_{i}, x_{i}\right)\right)^{\prime}\right]
$$

and let $\Omega^{*}$ denote the $(d+1) \times(d+1)$ matrix

$$
E\left[\left(\delta_{\alpha_{2}}^{*}\left(y_{i}, x_{i}, z_{i}\right)-\delta_{\alpha_{1}}^{*}\left(y_{i}, x_{i}, z_{i}\right)\right)\left(\delta_{\alpha_{2}}^{*}\left(y_{i}, x_{i}, z_{i}\right)-\delta_{\alpha_{1}}^{*}\left(y_{i}, x_{i}, z_{i}\right)\right)^{\prime}\right] ;
$$

then

$$
\sqrt{n}\left(\widehat{\beta}-\beta_{0}\right) \Rightarrow \mathrm{N}\left(0, J^{-1} \Omega J^{-1}\right)
$$

and

$$
\sqrt{n}\left(\hat{\gamma}-\gamma_{0}\right) \Rightarrow \mathrm{N}\left(0,\left(J^{*}\right)^{-1} \Omega^{*}\left(J^{*}\right)^{-1}\right) .
$$


For purposes of inference, we propose a consistent estimator for the limiting covariance matrices above. In both cases, the 'outer score' term involves the conditional density function of the residual. For the estimator under normality, this does not pose a problem, as the residual density is proportional to the standard normal density. All that is required is an estimator of the unknown scale function. For observations for which the weighting function is positive, the scale function can be easily estimated as

$$
\hat{\sigma}\left(x_{i}\right)=\frac{c_{\alpha_{2}}^{Z}\left(\hat{q}_{\alpha_{1}}\left(x_{i}\right)-x_{i}^{\prime} \hat{\beta}\right)+c_{\alpha_{1}}^{Z}\left(\hat{q}_{\alpha_{2}}\left(x_{i}\right)-x_{i}^{\prime} \hat{\beta}\right)}{2 c_{\alpha_{1}}^{Z} c_{\alpha_{2}}^{Z}} .
$$

For the estimator without normality, the 'outer score' term requires nonparametric density estimation. For this we propose a Nadaraya-Watson kernel estimator using the estimated residuals $\hat{v}_{j i}=y_{i}-\hat{q}_{\alpha_{j}}\left(x_{i}\right), i=1,2, \ldots, n, j=1,2$ :

$$
\hat{f}_{v_{j} \mid X}\left(0 \mid x_{i}\right)=\frac{\sum_{k \neq i} K_{h_{1 n}}^{(1)}\left(x_{k}-x_{i}\right) K_{h_{2 n}}^{(2)}\left(\hat{v}_{j k}\right)}{\sum_{k \neq i} K_{h_{1 n}}^{(1)}\left(x_{k}-x_{i}\right)},
$$

where $K^{(1)}$ and $K^{(2)}$, are continuously differentiable kernel functions on compact subsets of $\mathbb{R}^{d}$ and $\mathbb{R}$ respectively, that are positive, symmetric about 0 , and integrate to $1 . K_{h_{1 n}}^{(1)}(\cdot)$ and $K_{h_{2 n}}^{(2)}(\cdot)$ denote, respectively, $\hbar_{1 n}^{-d_{c}} K^{(1)}\left(\cdot / \hbar_{1 n}\right)$ and $\hbar_{2 n}^{-1} K^{(2)}\left(\cdot / \hbar_{2 n}\right)$, where $\hbar_{1 n}$ and $\hbar_{2 n}$ are bandwidth sequences which satisfy

1. $\hbar_{1 n}=\mathrm{o}(1), \hbar_{2 n}=\mathrm{o}(1)$.

2. $n \hbar_{1 n}^{d} \rightarrow \infty, n^{1 / 8} \hbar_{2 n} \rightarrow \infty$.

Using the scale function estimator and the kernel estimator for the conditional density, the following theorem proposes an estimator for each limiting covariance matrix and establishes its consistency. Again, we leave the proof to Appendix A.

Theorem 2. Define $\widehat{J}$ and $\hat{J}^{*}$ by the matrices

$$
\frac{1}{n} \sum_{i=1}^{n} \tau\left(x_{i}\right) \omega\left(\hat{q}_{\alpha_{1}}\left(x_{i}\right)\right) x_{i} x_{i}^{\prime}
$$

and

$$
\frac{1}{n} \sum_{i=1}^{n} \tau\left(x_{i}\right) \omega\left(\hat{q}_{\alpha_{1}}\left(x_{i}\right)\right) \hat{z}_{i} \hat{z}_{i}^{\prime}
$$

respectively. Let $\phi_{Z}(\cdot)$ denote the density function of the standard normal distribution, and let $\hat{\mathscr{V}}_{\alpha_{j}, i}, j=1,2$, be defined by

$$
\left(\frac{\bar{c}^{Z}}{\Delta c^{Z}}+\frac{(-1)^{j}}{2}\right) \tau\left(x_{i}\right) \omega\left(\hat{q}_{\alpha_{1}}\left(x_{i}\right)\right) \hat{\sigma}\left(x_{i}\right) \phi_{Z}\left(c_{\alpha_{j}}^{Z}\right)^{-1}
$$


and let $\hat{\mathscr{V}}_{\alpha_{j}, i}^{*}, j=1,2$, be defined by

$$
\left(\hat{\gamma}_{1}+\frac{(-1)^{j}}{2}\right) \tau\left(x_{i}\right) \omega\left(\hat{q}_{\alpha_{1}}\left(x_{i}\right)\right) \hat{f}_{v_{j} \mid X}^{-1}\left(0 \mid x_{i}\right)
$$

so that $\hat{\Omega}$ and $\hat{\Omega}^{*}$ can be defined as

$$
\begin{gathered}
\frac{1}{n} \sum_{i=1}^{n}\left(\hat{\mathscr{V}}_{\alpha_{2}, i}^{2}\left(\alpha_{2}\left(1-\alpha_{2}\right)\right)+\widehat{\mathscr{V}}_{\alpha_{1}, i}^{2}\left(\alpha_{1}\left(1-\alpha_{1}\right)\right)\right. \\
\left.-2 \hat{\mathscr{V}}_{\alpha_{2}, i} \hat{\mathscr{V}}_{\alpha_{1}, i}\left(\alpha_{1}\left(1-\alpha_{2}\right)\right)\right) x_{i} x_{i}^{\prime}
\end{gathered}
$$

and

$$
\frac{1}{n} \sum_{i=1}^{n}\left(\hat{\mathscr{V}}_{\alpha_{2}, i}^{*^{2}}\left(\alpha_{2}\left(1-\alpha_{2}\right)\right)+\hat{\mathscr{V}}_{\alpha_{1}, i}^{*^{2}}\left(\alpha_{1}\left(1-\alpha_{1}\right)\right)-2 \hat{\mathscr{V}}_{\alpha_{2}, i}^{*} \hat{\mathscr{V}}_{\alpha_{1}, i}^{*}\left(\alpha_{1}\left(1-\alpha_{2}\right)\right)\right) \hat{z}_{i} \hat{z}_{i}^{\prime}
$$

respectively; then

$$
\widehat{J} \stackrel{\mathrm{p}}{\rightarrow} J, \quad \hat{\Omega} \stackrel{\mathrm{p}}{\rightarrow} \Omega
$$

and

$$
\hat{J}^{*} \stackrel{p}{\rightarrow} J^{*}, \quad \hat{\Omega}^{*} \stackrel{\mathrm{p}}{\rightarrow} \Omega^{*}
$$

\section{Monte Carlo results}

In this section, the finite sample properties of the proposed estimators are examined through the results of a small-scale simulation study. In the study we consider various designs, with varying degrees of censoring, and compute basic summary statistics for the two estimators we introduce in this paper, referred to in this section as WNQN (weighted nonparametric quantile regression with normal errors) and WNQ (weighted nonparametric quantile regression), as well as other estimators for the censored regression model. These results are reported in Tables $1-18$.

We simulated from models of the form

$$
y_{i}=\max \left(a+x_{i} \beta_{0}+\sigma\left(x_{i}\right) u_{i}, 0\right),
$$

where $x_{i}$ was a random variable distributed standard normal, $\beta_{0}$ was set to 0.5 , and the error $\sigma\left(x_{i}\right) u_{i}$ varied to allow for four different designs:

1. homoskedastic normal: $\sigma\left(x_{i}\right) \equiv 1, u_{i} \sim$ standard normal;

2. homoskedastic Laplace: $\sigma\left(x_{i}\right) \equiv 1, u_{i} \sim$ Laplace;

3. heteroskedastic normal: $\sigma\left(x_{i}\right)=\mathscr{C} \exp \left(0.4 x_{i}^{2}\right), u_{i} \sim$ standard normal, and $\mathscr{C}$ was chosen so that the average value of the scale function was 1 ;

4. heteroskedastic Laplace: $\sigma\left(x_{i}\right)=\mathscr{C} \exp \left(0.4 x_{i}^{2}\right), u_{i} \sim$ Laplace. 
Table 1

Parametric estimators: homoskedastic design - normal errors

\begin{tabular}{|c|c|c|c|c|c|c|c|c|}
\hline & \multicolumn{2}{|c|}{$25 \%$ cens. } & \multicolumn{2}{|c|}{$40 \%$ cens. } & \multicolumn{2}{|l|}{$55 \%$ cens. } & \multicolumn{2}{|l|}{$65 \%$ cens. } \\
\hline & TOB1 & ТOB2 & TOB1 & ТОВ2 & TOB1 & ТOB2 & TOB1 & TOB2 \\
\hline \multicolumn{9}{|l|}{$n=100$} \\
\hline Mean bias & 0.0006 & 0.0005 & 0.0001 & 0.0006 & -0.0001 & 0.0021 & 0.0057 & 0.0067 \\
\hline Med. bias & -0.0032 & -0.0051 & -0.0018 & -0.0037 & -0.0871 & -0.0265 & -0.1089 & 0.0225 \\
\hline RMSE & 0.1066 & 0.1056 & 0.1149 & 0.1138 & 0.1245 & 0.1224 & 0.1438 & 0.1359 \\
\hline MAD & 0.0854 & 0.0846 & 0.0933 & 0.0919 & 0.0999 & 0.0969 & 0.1124 & 0.1080 \\
\hline \multicolumn{9}{|l|}{$n=200$} \\
\hline Mean bias & 0.0023 & 0.0028 & 0.0046 & 0.0060 & 0.0051 & 0.0077 & 0.0037 & 0.0080 \\
\hline Med. bias & -0.0005 & 0.0031 & -0.0019 & 0.0044 & -0.0098 & -0.0051 & 0.0873 & -0.0991 \\
\hline RMSE & 0.0769 & 0.0760 & 0.0860 & 0.0840 & 0.0974 & 0.0930 & 0.1042 & 0.0991 \\
\hline MAD & 0.0618 & 0.0610 & 0.0700 & 0.0676 & 0.0793 & 0.0745 & 0.0817 & 0.0771 \\
\hline \multicolumn{9}{|l|}{$n=400$} \\
\hline Mean bias & 0.0003 & 0.0006 & 0.0019 & 0.0020 & 0.0052 & 0.0052 & 0.0063 & 0.0068 \\
\hline Med. bias & -0.0044 & -0.0040 & -0.0030 & -0.0030 & -0.0328 & -0.0048 & -0.0664 & 0.0090 \\
\hline RMSE & 0.0507 & 0.0507 & 0.0565 & 0.0553 & 0.0620 & 0.0603 & 0.0667 & 0.0645 \\
\hline MAD & 0.0408 & 0.0408 & 0.0456 & 0.0447 & 0.0500 & 0.0481 & 0.0547 & 0.0519 \\
\hline
\end{tabular}

Table 2

Parametric estimators: homoskedastic design - Laplace errors

\begin{tabular}{|c|c|c|c|c|c|c|c|c|}
\hline & \multicolumn{2}{|c|}{$25 \%$ cens. } & \multicolumn{2}{|c|}{$40 \%$ cens. } & \multicolumn{2}{|l|}{$55 \%$ cens. } & \multicolumn{2}{|c|}{$65 \%$ cens. } \\
\hline & TOB1 & ТOB2 & TOB1 & TOB2 & TOB1 & ТОВ2 & TOB1 & ТOB2 \\
\hline \multicolumn{9}{|l|}{$n=100$} \\
\hline Mean bias & 0.0144 & -0.0227 & 0.0569 & -0.0159 & 0.0861 & -0.0280 & 0.1026 & -0.0476 \\
\hline Med. bias & 0.0126 & -0.0222 & 0.0504 & -0.0203 & -0.0188 & -0.0342 & -0.0245 & 0.0626 \\
\hline RMSE & 0.1429 & 0.1337 & 0.1775 & 0.1448 & 0.2078 & 0.1560 & 0.2396 & 0.1696 \\
\hline MAD & 0.1118 & 0.1054 & 0.1326 & 0.1124 & 0.1565 & 0.1222 & 0.1805 & 0.1358 \\
\hline \multicolumn{9}{|l|}{$n=200$} \\
\hline Mean bias & 0.0217 & -0.0179 & 0.0624 & -0.0140 & 0.0984 & -0.0246 & 0.1194 & -0.0441 \\
\hline Med. bias & 0.0168 & -0.0199 & 0.0601 & -0.0168 & 0.0512 & -0.0052 & 0.0706 & -0.0061 \\
\hline RMSE & 0.1014 & 0.0932 & 0.1307 & 0.0995 & 0.1690 & 0.1102 & 0.2027 & 0.1245 \\
\hline MAD & 0.0821 & 0.0757 & 0.1040 & 0.0804 & 0.1321 & 0.0884 & 0.1559 & 0.1005 \\
\hline \multicolumn{9}{|l|}{$n=400$} \\
\hline Mean bias & 0.0173 & -0.0220 & 0.0592 & -0.0177 & 0.0939 & -0.0299 & 0.1105 & -0.0509 \\
\hline Med. bias & 0.0160 & -0.0241 & 0.0594 & -0.0181 & -0.0287 & -0.0342 & 0.1326 & 0.0255 \\
\hline RMSE & 0.0737 & 0.0693 & 0.0992 & 0.0713 & 0.1320 & 0.0800 & 0.1530 & 0.0936 \\
\hline MAD & 0.0591 & 0.0562 & 0.0802 & 0.0575 & 0.1079 & 0.0650 & 0.1253 & 0.0768 \\
\hline
\end{tabular}


Table 3

Parametric estimators: heteroskedastic design - normal errors

\begin{tabular}{|c|c|c|c|c|c|c|c|c|}
\hline & \multicolumn{2}{|c|}{$25 \%$ cens. } & \multicolumn{2}{|c|}{$40 \%$ cens. } & \multicolumn{2}{|c|}{$55 \%$ cens. } & \multicolumn{2}{|c|}{$65 \%$ cens. } \\
\hline & TOB1 & TOB2 & TOB1 & TOB2 & TOB1 & TOB2 & TOB1 & TOB2 \\
\hline \multicolumn{9}{|l|}{$n=100$} \\
\hline Mean bias & 0.2420 & 0.0002 & 0.2019 & -0.0017 & 0.2895 & 0.0011 & 0.4081 & 0.0028 \\
\hline Med. bias & -0.0649 & 0.0022 & -0.0438 & -0.0001 & -0.0591 & 0.0399 & -0.0650 & 0.0048 \\
\hline RMSE & 3.6775 & 0.1049 & 1.6223 & 0.1115 & 1.8980 & 0.1224 & 2.3233 & 0.1314 \\
\hline MAD & 0.6884 & 0.0852 & 0.5531 & 0.0908 & 0.6117 & 0.1000 & 0.7195 & 0.1064 \\
\hline \multicolumn{9}{|l|}{$n=200$} \\
\hline Mean bias & 0.1431 & -0.0037 & 0.2406 & -0.0026 & 0.3785 & -0.0018 & 0.4906 & -0.0019 \\
\hline Med. bias & -0.0426 & -0.0063 & -0.0078 & -0.0060 & -0.0434 & -0.0202 & -0.0087 & 0.0302 \\
\hline RMSE & 1.4950 & 0.0767 & 1.9803 & 0.0809 & 2.5594 & 0.0866 & 2.9190 & 0.0898 \\
\hline MAD & 0.3589 & 0.0617 & 0.4157 & 0.0654 & 0.5138 & 0.0687 & 0.6067 & 0.0717 \\
\hline \multicolumn{9}{|l|}{$n=400$} \\
\hline Mean bias & 0.1063 & 0.0010 & 0.2069 & 0.0024 & 0.3654 & 0.0035 & 0.5061 & 0.0065 \\
\hline Med. bias & -0.0039 & 0.0018 & 0.0398 & 0.0004 & 0.0475 & -0.0035 & 0.0533 & -0.0462 \\
\hline RMSE & 0.7066 & 0.0540 & 0.8459 & 0.0588 & 1.1458 & 0.0623 & 1.4067 & 0.0668 \\
\hline MAD & 0.2868 & 0.0434 & 0.3228 & 0.0469 & 0.4271 & 0.0500 & 0.5444 & 0.0539 \\
\hline
\end{tabular}

Table 4

Parametric estimators: heteroskedastic design - Laplace errors

\begin{tabular}{|c|c|c|c|c|c|c|c|c|}
\hline & \multicolumn{2}{|c|}{$25 \%$ cens. } & \multicolumn{2}{|c|}{$40 \%$ cens. } & \multicolumn{2}{|c|}{$55 \%$ cens. } & \multicolumn{2}{|c|}{$65 \%$ cens. } \\
\hline & ТOB1 & ТOB2 & TOB1 & ТОВ2 & TOB1 & ТOB2 & TOB1 & ТОВ2 \\
\hline \multicolumn{9}{|l|}{$n=100$} \\
\hline Mean bias & 0.0148 & -0.0147 & 0.1395 & -0.0022 & 0.3217 & -0.0109 & 0.3870 & -0.0330 \\
\hline Med. bias & -0.0251 & -0.0138 & 0.0212 & -0.0016 & -0.1849 & -0.0417 & 0.0314 & -0.0587 \\
\hline RMSE & 1.4794 & 0.1331 & 1.4050 & 0.1417 & 1.6714 & 0.1537 & 1.5995 & 0.1709 \\
\hline MAD & 0.5073 & 0.1049 & 0.5353 & 0.1113 & 0.6393 & 0.1206 & 0.6530 & 0.1358 \\
\hline \multicolumn{9}{|l|}{$n=200$} \\
\hline Mean bias & 0.1645 & -0.0233 & 0.2996 & -0.0162 & 0.4719 & -0.0273 & 0.5909 & -0.0520 \\
\hline Med. bias & -0.0037 & -0.0259 & 0.0654 & -0.0227 & -0.1252 & 0.0287 & 0.0887 & -0.0268 \\
\hline RMSE & 1.2557 & 0.0997 & 1.4861 & 0.1030 & 1.7876 & 0.1115 & 2.0462 & 0.1253 \\
\hline MAD & 0.4582 & 0.0803 & 0.5144 & 0.0833 & 0.6137 & 0.0897 & 0.7071 & 0.1010 \\
\hline \multicolumn{9}{|l|}{$n=400$} \\
\hline Mean bias & 0.2811 & -0.0159 & 0.8530 & -0.0082 & 1.5325 & -0.0214 & 1.6841 & -0.0461 \\
\hline Med. bias & 0.0206 & -0.0164 & 0.1061 & -0.0089 & -0.0059 & -0.0483 & 0.1988 & -0.0599 \\
\hline RMSE & 14.1494 & 0.0670 & 13.9385 & 0.0673 & 15.3438 & 0.0756 & 17.1026 & 0.0883 \\
\hline MAD & 1.4257 & 0.0531 & 1.3709 & 0.0530 & 1.5681 & 0.0603 & 1.7047 & 0.0724 \\
\hline
\end{tabular}


Table 5

Parametric estimators: heteroskedastic design - normal errors

\begin{tabular}{|c|c|c|c|c|c|c|c|c|}
\hline & \multicolumn{2}{|l|}{$25 \%$ cens. } & \multicolumn{2}{|c|}{$40 \%$ cens. } & \multicolumn{2}{|l|}{$55 \%$ cens. } & \multicolumn{2}{|l|}{$65 \%$ cens. } \\
\hline & TOB3 & TOB4 & TOB3 & TOB4 & TOB3 & TOB4 & TOB3 & TOB4 \\
\hline \multicolumn{9}{|l|}{$n=100$} \\
\hline Mean bias & -0.1350 & 0.3256 & -0.1724 & 0.4399 & -0.2085 & 0.5505 & -0.2363 & 0.6062 \\
\hline Med. bias & -0.2189 & 0.3085 & -0.2603 & 0.4304 & -0.2108 & 0.5032 & -0.2534 & 0.2466 \\
\hline RMSE & 1.5903 & 0.3822 & 1.5963 & 0.4943 & 1.6022 & 0.6190 & 1.6073 & 0.6869 \\
\hline MAD & 0.6920 & 0.3330 & 0.7129 & 0.4442 & 0.7345 & 0.5548 & 0.7512 & 0.6102 \\
\hline \multicolumn{9}{|l|}{$n=200$} \\
\hline Mean bias & -0.1400 & 0.3256 & -0.1772 & 0.4601 & -0.2129 & 0.5681 & -0.2410 & 0.6318 \\
\hline Med. bias & -0.1870 & 0.3182 & -0.2225 & 0.4726 & -0.1769 & 0.2963 & -0.2280 & 0.4075 \\
\hline RMSE & 1.2495 & 0.3541 & 1.2552 & 0.4917 & 1.2615 & 0.6013 & 1.2669 & 0.6702 \\
\hline MAD & 0.5444 & 0.3257 & 0.5644 & 0.4601 & 0.5870 & 0.5681 & 0.6061 & 0.6318 \\
\hline \multicolumn{9}{|l|}{$n=400$} \\
\hline Mean bias & -0.2991 & 0.3261 & -0.3332 & 0.4545 & -0.3657 & 0.5430 & -0.3913 & 0.6057 \\
\hline Med. bias & -0.2091 & 0.3163 & -0.2476 & 0.4469 & -0.2229 & 0.3975 & -0.2353 & 0.4335 \\
\hline RMSE & 0.7037 & 0.3411 & 0.7178 & 0.4715 & 0.7324 & 0.5562 & 0.7449 & 0.6240 \\
\hline MAD & 0.4437 & 0.3261 & 0.4638 & 0.4545 & 0.4835 & 0.5430 & 0.4990 & 0.6057 \\
\hline
\end{tabular}

Table 6

Parametric estimators: heteroskedastic design - Laplace errors

\begin{tabular}{|c|c|c|c|c|c|c|c|c|}
\hline & \multicolumn{2}{|l|}{$25 \%$ cens. } & \multicolumn{2}{|c|}{$40 \%$ cens. } & \multicolumn{2}{|c|}{$55 \%$ cens. } & \multicolumn{2}{|l|}{$65 \%$ cens. } \\
\hline & TOB3 & TOB4 & ТOB3 & TOB4 & ТОВ3 & TOB4 & TOB3 & TOB4 \\
\hline \multicolumn{9}{|l|}{$n=100$} \\
\hline Mean bias & -0.3057 & 0.2598 & -0.3419 & 0.4247 & -0.3783 & 0.5563 & -0.4062 & 0.5238 \\
\hline Med. bias & -0.1912 & 0.2713 & -0.2307 & 0.4436 & -0.1792 & 0.4729 & -0.2613 & 0.4391 \\
\hline RMSE & 1.2457 & 0.3371 & 1.2555 & 0.4977 & 1.2665 & 0.6325 & 1.2755 & 0.6167 \\
\hline MAD & 0.6335 & 0.2844 & 0.6482 & 0.4398 & 0.6659 & 0.5615 & 0.6823 & 0.5326 \\
\hline \multicolumn{9}{|l|}{$n=200$} \\
\hline Mean bias & -0.0754 & 0.2768 & -0.1101 & 0.4594 & -0.1448 & 0.5531 & -0.1714 & 0.5152 \\
\hline Med. bias & -0.2673 & 0.2487 & -0.3075 & 0.4593 & -0.2673 & 0.4079 & -0.3078 & 0.7197 \\
\hline RMSE & 1.4226 & 0.3207 & 1.4284 & 0.4922 & 1.4336 & 0.5829 & 1.4374 & 0.5566 \\
\hline MAD & 0.7159 & 0.2834 & 0.7379 & 0.4594 & 0.7603 & 0.5531 & 0.7774 & 0.5152 \\
\hline \multicolumn{9}{|l|}{$n=400$} \\
\hline Mean bias & -0.2651 & 0.2687 & -0.2993 & 0.4376 & -0.3328 & 0.5300 & -0.3595 & 0.5075 \\
\hline Med. bias & -0.2459 & 0.2841 & -0.2820 & 0.4440 & -0.2459 & 0.2375 & -0.2820 & 0.3505 \\
\hline RMSE & 0.9222 & 0.2982 & 0.9308 & 0.4587 & 0.9409 & 0.5566 & 0.9494 & 0.5396 \\
\hline MAD & 0.5098 & 0.2704 & 0.5243 & 0.4376 & 0.5414 & 0.5300 & 0.5572 & 0.5075 \\
\hline
\end{tabular}


Table 7

Quantile regression estimators: homoskedastic design - normal errors

\begin{tabular}{|c|c|c|c|c|c|c|c|c|}
\hline & \multicolumn{2}{|c|}{$25 \%$ cens. } & \multicolumn{2}{|c|}{$40 \%$ cens. } & \multicolumn{2}{|c|}{$55 \%$ cens. } & \multicolumn{2}{|l|}{$65 \%$ cens. } \\
\hline & $\alpha=0.5$ & $\alpha=0.75$ & $\alpha=0.5$ & $\alpha=0.75$ & $\alpha=0.5$ & $\alpha=0.75$ & $\alpha=0.5$ & $\alpha=0.75$ \\
\hline \multicolumn{9}{|l|}{$n=100$} \\
\hline Mean bias & 0.0002 & 0.0115 & -0.0340 & -0.0129 & -0.0760 & -0.0231 & -0.2700 & -0.0715 \\
\hline Med. bias & -0.0155 & 0.0165 & -0.0661 & -0.0258 & -0.1003 & -0.0453 & -0.4357 & -0.0887 \\
\hline RMSE & 0.1605 & 0.1377 & 0.2115 & 0.1364 & 0.2740 & 0.1590 & 0.4193 & 0.2029 \\
\hline MAD & 0.1212 & 0.1094 & 0.1641 & 0.1079 & 0.2191 & 0.1275 & 0.3657 & 0.1648 \\
\hline \multicolumn{9}{|l|}{$n=200$} \\
\hline Mean bias & -0.0115 & -0.0091 & -0.0106 & -0.0024 & -0.0356 & -0.0145 & -0.2261 & -0.0359 \\
\hline Med. bias & -0.0126 & -0.0080 & -0.0282 & -0.0077 & -0.0506 & -0.0364 & -0.3565 & -0.0614 \\
\hline RMSE & 0.1049 & 0.0936 & 0.1438 & 0.1022 & 0.2639 & 0.1234 & 0.4081 & 0.1646 \\
\hline MAD & 0.0808 & 0.0726 & 0.1135 & 0.0812 & 0.1947 & 0.0956 & 0.3622 & 0.1310 \\
\hline \multicolumn{9}{|l|}{$n=400$} \\
\hline Mean bias & -0.0044 & 0.0000 & -0.0229 & -0.0105 & -0.0299 & -0.0200 & -0.2392 & -0.0125 \\
\hline Med. bias & -0.0018 & -0.0040 & -0.0246 & -0.0088 & -0.0515 & -0.0227 & -0.3150 & -0.0198 \\
\hline RMSE & 0.0683 & 0.0676 & 0.0960 & 0.0704 & 0.1750 & 0.0952 & 0.3856 & 0.1155 \\
\hline MAD & 0.0549 & 0.0547 & 0.0764 & 0.0579 & 0.1345 & 0.0761 & 0.3311 & 0.0905 \\
\hline
\end{tabular}

Table 8

Quantile regression estimators: homoskedastic design - Laplace errors

\begin{tabular}{|c|c|c|c|c|c|c|c|c|}
\hline & \multicolumn{2}{|c|}{$25 \%$ cens. } & \multicolumn{2}{|c|}{$40 \%$ cens. } & \multicolumn{2}{|c|}{$55 \%$ cens. } & \multicolumn{2}{|l|}{$65 \%$ cens. } \\
\hline & $\alpha=0.5$ & $\alpha=0.75$ & $\alpha=0.5$ & $\alpha=0.75$ & $\alpha=0.5$ & $\alpha=0.75$ & $\alpha=0.5$ & $\alpha=0.75$ \\
\hline \multicolumn{9}{|l|}{$n=100$} \\
\hline Mean bias & -0.0033 & 0.0042 & -0.0229 & -0.0025 & -0.0505 & -0.0221 & -0.2337 & -0.0733 \\
\hline Med. bias & -0.0145 & 0.0049 & -0.0396 & -0.0177 & -0.0959 & -0.0467 & -0.3540 & -0.1229 \\
\hline RMSE & 0.1384 & 0.1890 & 0.1752 & 0.1718 & 0.3233 & 0.2168 & 0.4859 & 0.2409 \\
\hline MAD & 0.1049 & 0.1430 & 0.1377 & 0.1315 & 0.2310 & 0.1644 & 0.3761 & 0.1986 \\
\hline \multicolumn{9}{|l|}{$n=200$} \\
\hline Mean bias & -0.0116 & -0.0129 & -0.0147 & -0.0122 & -0.0349 & -0.0311 & -0.1980 & -0.0167 \\
\hline Med. bias & -0.0174 & -0.0201 & -0.0272 & -0.0082 & -0.0602 & -0.0481 & -0.2618 & -0.0533 \\
\hline RMSE & 0.0872 & 0.1186 & 0.1391 & 0.1202 & 0.2474 & 0.1427 & 0.4019 & 0.1976 \\
\hline MAD & 0.0690 & 0.0964 & 0.1065 & 0.0959 & 0.1791 & 0.1161 & 0.3440 & 0.1516 \\
\hline \multicolumn{9}{|l|}{$n=400$} \\
\hline Mean bias & -0.0031 & -0.0083 & -0.0118 & -0.0156 & -0.0005 & -0.0094 & -0.1741 & -0.0308 \\
\hline Med. bias & -0.0060 & -0.0119 & -0.0164 & -0.0188 & -0.0135 & -0.0247 & -0.1942 & -0.0512 \\
\hline RMSE & 0.0628 & 0.0873 & 0.0921 & 0.0871 & 0.1673 & 0.1142 & 0.3578 & 0.1380 \\
\hline MAD & 0.0485 & 0.0686 & 0.0736 & 0.0691 & 0.1219 & 0.0892 & 0.3016 & 0.1122 \\
\hline
\end{tabular}


Table 9

Quantile regression estimators: heteroskedastic design - normal errors

\begin{tabular}{|c|c|c|c|c|c|c|c|c|}
\hline & \multicolumn{2}{|l|}{$25 \%$ cens. } & \multicolumn{2}{|c|}{$40 \%$ cens. } & \multicolumn{2}{|l|}{$55 \%$ cens. } & \multicolumn{2}{|c|}{$65 \%$ cens. } \\
\hline & $\alpha=0.5$ & $\alpha=0.75$ & $\alpha=0.5$ & $\alpha=0.75$ & $\alpha=0.5$ & $\alpha=0.75$ & $\alpha=0.5$ & $\alpha=0.75$ \\
\hline \multicolumn{9}{|l|}{$n=100$} \\
\hline Mean bias & -0.0009 & 0.0610 & 0.0117 & 0.1257 & 0.2149 & -0.1270 & 0.2888 & 1.1028 \\
\hline Med. bias & -0.0185 & 0.0205 & -0.0545 & 0.0660 & -0.0795 & 0.2291 & -0.3454 & 0.3264 \\
\hline RMSE & 0.1958 & 0.2759 & 0.2746 & 0.3549 & 1.6838 & 10.3271 & 3.3456 & 4.8252 \\
\hline MAD & 0.1415 & 0.2024 & 0.2010 & 0.2468 & 0.5407 & 1.1712 & 0.8425 & 1.2173 \\
\hline \multicolumn{9}{|l|}{$n=200$} \\
\hline Mean bias & -0.0061 & 0.0353 & 0.0069 & 0.1516 & 0.1004 & 0.4391 & 0.1805 & 0.3670 \\
\hline Med. bias & -0.0163 & 0.0335 & -0.0265 & 0.1197 & -0.0416 & 0.3034 & -0.2829 & 0.4113 \\
\hline RMSE & 0.1259 & 0.1696 & 0.1941 & 0.2917 & 0.6586 & 0.9800 & 1.8916 & 12.2984 \\
\hline MAD & 0.0970 & 0.1363 & 0.1395 & 0.2055 & 0.3398 & 0.4573 & 0.7017 & 1.8711 \\
\hline \multicolumn{9}{|l|}{$n=400$} \\
\hline Mean bias & -0.0100 & 0.0588 & 0.0030 & 0.1847 & 0.0375 & 0.4053 & 0.2082 & 0.6775 \\
\hline Med. bias & -0.0164 & 0.0650 & -0.0127 & 0.1759 & -0.0415 & 0.3210 & -0.2222 & 0.5964 \\
\hline RMSE & 0.0876 & 0.1415 & 0.1279 & 0.2368 & 0.4463 & 0.5383 & 2.7996 & 0.8694 \\
\hline MAD & 0.0683 & 0.1150 & 0.0965 & 0.1968 & 0.2497 & 0.4066 & 0.6979 & 0.6934 \\
\hline
\end{tabular}

Table 10

Quantile regression estimators: heteroskedastic design - Laplace errors

\begin{tabular}{|c|c|c|c|c|c|c|c|c|}
\hline & \multicolumn{2}{|c|}{$25 \%$ cens. } & \multicolumn{2}{|c|}{$40 \%$ cens. } & \multicolumn{2}{|c|}{$55 \%$ cens. } & \multicolumn{2}{|c|}{$65 \%$ cens. } \\
\hline & $\alpha=0.5$ & $\alpha=0.75$ & $\alpha=0.5$ & $\alpha=0.75$ & $\alpha=0.5$ & $\alpha=0.75$ & $\alpha=0.5$ & $\alpha=0.75$ \\
\hline \multicolumn{9}{|l|}{$n=100$} \\
\hline Mean bias & -0.0001 & 0.0418 & 0.0204 & 0.1701 & 0.0888 & -0.6106 & 0.3059 & -10.4319 \\
\hline Med. bias & -0.0197 & -0.0019 & -0.0272 & 0.0580 & -0.0587 & 0.1668 & -0.2934 & 0.2388 \\
\hline RMSE & 0.1615 & 0.2875 & 0.3775 & 0.5519 & 4.1080 & 16.0690 & 3.0633 & 166.9228 \\
\hline MAD & 0.1230 & 0.2116 & 0.1874 & 0.3029 & 0.7781 & 1.9788 & 0.8324 & 12.0269 \\
\hline \multicolumn{9}{|l|}{$n=200$} \\
\hline Mean bias & -0.0100 & 0.0321 & -0.0054 & 0.1510 & 0.1254 & -0.7184 & 1.8759 & 1.8039 \\
\hline Med. bias & -0.0183 & 0.0171 & -0.0372 & 0.1434 & -0.0431 & 0.2701 & -0.2185 & 0.4701 \\
\hline RMSE & 0.1053 & 0.1920 & 0.1669 & 0.3036 & 1.7076 & 19.0253 & 29.5495 & 24.5472 \\
\hline MAD & 0.0830 & 0.1488 & 0.1262 & 0.2347 & 0.3575 & 1.5174 & 2.3717 & 2.5987 \\
\hline \multicolumn{9}{|l|}{$n=400$} \\
\hline Mean bias & -0.0021 & 0.0358 & -0.0043 & 0.1633 & 0.0350 & 0.4185 & 0.0228 & -0.4632 \\
\hline Med. bias & -0.0007 & 0.0304 & -0.0221 & 0.1513 & -0.0015 & 0.3344 & -0.2075 & 0.5179 \\
\hline RMSE & 0.0683 & 0.1380 & 0.1175 & 0.2447 & 0.2607 & 0.5421 & 1.2718 & 20.9197 \\
\hline MAD & 0.0533 & 0.1068 & 0.0919 & 0.1970 & 0.1676 & 0.4280 & 0.5056 & 1.9593 \\
\hline
\end{tabular}


Table 11

WNQ estimator: homoskedastic design - normal errors

\begin{tabular}{|c|c|c|c|c|c|c|c|c|}
\hline & \multicolumn{2}{|l|}{$25 \%$ cens. } & \multicolumn{2}{|l|}{$40 \%$ cens. } & \multicolumn{2}{|l|}{$55 \%$ cens. } & \multicolumn{2}{|l|}{$65 \%$ cens. } \\
\hline & $\varepsilon=0.025$ & $\varepsilon=0.05$ & $\varepsilon=0.025$ & $\varepsilon=0.05$ & $\varepsilon=0.025$ & $\varepsilon=0.05$ & $\varepsilon=0.025$ & $\varepsilon=0.05$ \\
\hline \multicolumn{9}{|l|}{$n=100$} \\
\hline Mean bias & -0.0673 & -0.0876 & -0.1226 & -0.0950 & -0.1202 & -0.1072 & -0.2327 & -0.2240 \\
\hline Med. bias & -0.0627 & -0.0885 & -0.1111 & -0.1022 & -0.1013 & -0.1273 & -0.1838 & -0.2513 \\
\hline RMSE & 0.1783 & 0.1844 & 0.2396 & 0.2130 & 0.2994 & 0.2648 & 0.5990 & 0.5776 \\
\hline MAD & 0.1399 & 0.1480 & 0.1830 & 0.1709 & 0.2246 & 0.2115 & 0.4285 & 0.4367 \\
\hline \multicolumn{9}{|l|}{$n=200$} \\
\hline Mean bias & -0.0601 & -0.0671 & -0.0851 & -0.1113 & -0.1050 & 0.0412 & 0.0627 & -0.0905 \\
\hline Med. bias & -0.0590 & -0.0632 & -0.0855 & -0.1214 & -0.0992 & 0.0168 & 0.0971 & -0.0827 \\
\hline RMSE & 0.1241 & 0.1307 & 0.1463 & 0.1649 & 0.1819 & 0.1742 & 0.2632 & 0.2298 \\
\hline MAD & 0.0994 & 0.1025 & 0.1195 & 0.1363 & 0.1532 & 0.1441 & 0.2144 & 0.1905 \\
\hline \multicolumn{9}{|l|}{$n=400$} \\
\hline Mean bias & -0.0503 & -0.0496 & -0.0868 & -0.0937 & -0.0834 & -0.0228 & 0.0209 & -0.0759 \\
\hline Med. bias & -0.0538 & -0.0482 & -0.0870 & -0.0920 & -0.0934 & -0.0061 & 0.0071 & -0.1072 \\
\hline RMSE & 0.0864 & 0.0833 & 0.1161 & 0.1189 & 0.1475 & 0.1543 & 0.1635 & 0.1703 \\
\hline MAD & 0.0710 & 0.0681 & 0.0974 & 0.1006 & 0.1261 & 0.1323 & 0.1353 & 0.1425 \\
\hline
\end{tabular}

Table 12

WNQN estimator: homoskedastic design - normal errors

\begin{tabular}{|c|c|c|c|c|c|c|c|c|}
\hline & \multicolumn{2}{|l|}{$25 \%$ cens. } & \multicolumn{2}{|l|}{$40 \%$ cens. } & \multicolumn{2}{|l|}{$55 \%$ cens. } & \multicolumn{2}{|l|}{$65 \%$ cens. } \\
\hline & $\varepsilon=0.025$ & $\varepsilon=0.05$ & $\varepsilon=0.025$ & $\varepsilon=0.05$ & $\varepsilon=0.025$ & $\varepsilon=0.05$ & $\varepsilon=0.025$ & $\varepsilon=0.05$ \\
\hline \multicolumn{9}{|l|}{$n=100$} \\
\hline Mean bias & -0.0766 & -0.0742 & -0.1170 & -0.0996 & -0.1048 & -0.0818 & -0.2185 & -0.2731 \\
\hline Med. bias & -0.0863 & -0.0778 & -0.0864 & -0.0985 & -0.1222 & -0.0909 & -0.1194 & -0.2229 \\
\hline RMSE & 0.2611 & 0.2151 & 0.4268 & 0.3167 & 0.7187 & 0.4439 & 1.2727 & 1.1294 \\
\hline MAD & 0.2110 & 0.1718 & 0.3058 & 0.2396 & 0.5362 & 0.3459 & 0.8551 & 0.7018 \\
\hline \multicolumn{9}{|l|}{$n=200$} \\
\hline Mean bias & -0.0546 & -0.0681 & -0.1035 & -0.1205 & -0.1119 & -0.0929 & -0.1465 & -0.0783 \\
\hline Med. bias & -0.0463 & -0.0558 & -0.0927 & -0.1154 & -0.1159 & -0.0972 & -0.1223 & -0.0904 \\
\hline RMSE & 0.1534 & 0.1556 & 0.2387 & 0.2034 & 0.3291 & 0.2641 & 0.5706 & 0.3961 \\
\hline MAD & 0.1191 & 0.1228 & 0.1873 & 0.1594 & 0.2594 & 0.2140 & 0.4351 & 0.3079 \\
\hline \multicolumn{9}{|l|}{$n=400$} \\
\hline Mean bias & -0.0499 & -0.0495 & -0.0986 & -0.1097 & -0.0936 & -0.0888 & -0.1170 & -0.0722 \\
\hline Med. bias & -0.0538 & -0.0448 & -0.0899 & -0.0987 & -0.1070 & -0.1009 & -0.1093 & -0.0873 \\
\hline RMSE & 0.1085 & 0.0966 & 0.1675 & 0.1591 & 0.2337 & 0.2006 & 0.3604 & 0.2861 \\
\hline MAD & 0.0873 & 0.0762 & 0.1313 & 0.1290 & 0.1862 & 0.1624 & 0.2774 & 0.2256 \\
\hline
\end{tabular}


Table 13

WNQ estimator: homoskedastic design - Laplace errors

\begin{tabular}{|c|c|c|c|c|c|c|c|c|}
\hline & \multicolumn{2}{|l|}{$25 \%$ cens. } & \multicolumn{2}{|l|}{$40 \%$ cens. } & \multicolumn{2}{|c|}{$55 \%$ cens. } & \multicolumn{2}{|l|}{$65 \%$ cens. } \\
\hline & $\varepsilon=0.025$ & $\varepsilon=0.05$ & $\varepsilon=0.025$ & $\varepsilon=0.05$ & $\varepsilon=0.025$ & $\varepsilon=0.05$ & $\varepsilon=0.025$ & $\varepsilon=0.05$ \\
\hline \multicolumn{9}{|l|}{$n=100$} \\
\hline Mean bias & -0.0450 & -0.0582 & -0.0785 & -0.0628 & -0.1321 & -0.0862 & -0.2488 & -0.2638 \\
\hline Med. bias & -0.0659 & -0.0697 & -0.0916 & -0.0867 & -0.1367 & -0.0917 & -0.3168 & -0.2728 \\
\hline RMSE & 0.2318 & 0.2109 & 0.2769 & 0.3055 & 0.4119 & 0.4167 & 1.0671 & 1.0201 \\
\hline MAD & 0.1851 & 0.1688 & 0.2220 & 0.2434 & 0.3234 & 0.3050 & 0.7490 & 0.6516 \\
\hline \multicolumn{9}{|l|}{$n=200$} \\
\hline Mean bias & -0.0482 & -0.0546 & -0.0801 & -0.0880 & -0.1159 & -0.0391 & -0.1824 & -0.0395 \\
\hline Med. bias & -0.0431 & -0.0585 & -0.0814 & -0.0866 & -0.1251 & 0.1188 & -0.0792 & -0.0565 \\
\hline RMSE & 0.1364 & 0.1285 & 0.1701 & 0.1782 & 0.2314 & 0.2297 & 0.3973 & 0.3642 \\
\hline MAD & 0.1102 & 0.1026 & 0.1370 & 0.1423 & 0.1871 & 0.1930 & 0.3164 & 0.2917 \\
\hline \multicolumn{9}{|l|}{$n=400$} \\
\hline Mean bias & -0.0447 & -0.0395 & -0.0910 & -0.0942 & -0.1047 & -0.0354 & 0.0441 & -0.0333 \\
\hline Med. bias & -0.0473 & -0.0484 & -0.0920 & -0.0905 & -0.1072 & 0.0029 & 0.1015 & -0.0799 \\
\hline RMSE & 0.0917 & 0.0903 & 0.1283 & 0.1345 & 0.1795 & 0.1894 & 0.2723 & 0.2321 \\
\hline MAD & 0.0736 & 0.0745 & 0.1068 & 0.1121 & 0.1504 & 0.1624 & 0.2229 & 0.1839 \\
\hline
\end{tabular}

Table 14

WNQN estimator: homoskedastic design - Laplace errors

\begin{tabular}{|c|c|c|c|c|c|c|c|c|}
\hline & \multicolumn{2}{|l|}{$25 \%$ cens. } & \multicolumn{2}{|l|}{$40 \%$ cens. } & \multicolumn{2}{|l|}{$55 \%$ cens. } & \multicolumn{2}{|l|}{$65 \%$ cens. } \\
\hline & $\varepsilon=0.025$ & $\varepsilon=0.05$ & $\varepsilon=0.025$ & $\varepsilon=0.05$ & $\varepsilon=0.025$ & $\varepsilon=0.05$ & $\varepsilon=0.025$ & $\varepsilon=0.05$ \\
\hline \multicolumn{9}{|l|}{$n=100$} \\
\hline Mean bias & -0.0510 & -0.0685 & -0.0661 & -0.0840 & -0.1694 & -0.1633 & -0.1657 & 0.2858 \\
\hline Med. bias & -0.0524 & -0.0585 & -0.0520 & -0.1071 & -0.1693 & -0.0914 & -0.1344 & 0.1182 \\
\hline RMSE & 0.3051 & 0.2455 & 0.5254 & 0.4321 & 0.9538 & 0.7693 & 1.6101 & 0.8604 \\
\hline MAD & 0.2425 & 0.1882 & 0.3918 & 0.3367 & 0.6914 & 0.5762 & 1.3245 & 1.3538 \\
\hline \multicolumn{9}{|l|}{$n=200$} \\
\hline Mean bias & -0.0547 & -0.0598 & -0.1012 & -0.0943 & -0.1146 & -0.1298 & -0.1868 & -0.1420 \\
\hline Med. bias & -0.0639 & -0.0653 & -0.0856 & -0.0911 & -0.1236 & -0.1054 & -0.2086 & -0.1531 \\
\hline RMSE & 0.1793 & 0.1481 & 0.3004 & 0.2267 & 0.4555 & 0.3664 & 0.8237 & 0.5705 \\
\hline MAD & 0.1445 & 0.1163 & 0.2261 & 0.1786 & 0.3374 & 0.2809 & 0.6270 & 0.4512 \\
\hline \multicolumn{9}{|l|}{$n=400$} \\
\hline Mean bias & -0.0464 & -0.0412 & -0.0849 & -0.0885 & -0.1047 & -0.0961 & -0.1064 & -0.0692 \\
\hline Med. bias & -0.0492 & -0.0477 & -0.0774 & -0.0907 & -0.1082 & -0.0987 & -0.1169 & -0.0668 \\
\hline RMSE & 0.1140 & 0.1008 & 0.2058 & 0.1609 & 0.2760 & 0.2616 & 0.4859 & 0.3711 \\
\hline MAD & 0.0907 & 0.0821 & 0.1617 & 0.1285 & 0.2270 & 0.2110 & 0.3872 & 0.2873 \\
\hline
\end{tabular}


Table 15

WNQ estimator: heteroskedastic design - normal errors

\begin{tabular}{|c|c|c|c|c|c|c|c|c|}
\hline & \multicolumn{2}{|l|}{$25 \%$ cens. } & \multicolumn{2}{|l|}{$40 \%$ cens. } & \multicolumn{2}{|c|}{$55 \%$ cens. } & \multicolumn{2}{|l|}{$65 \%$ cens. } \\
\hline & $\varepsilon=0.025$ & $\varepsilon=0.05$ & $\varepsilon=0.025$ & $\varepsilon=0.05$ & $\varepsilon=0.025$ & $\varepsilon=0.05$ & $\varepsilon=0.025$ & $\varepsilon=0.05$ \\
\hline \multicolumn{9}{|l|}{$n=100$} \\
\hline Mean bias & -0.0213 & -0.0301 & -0.0224 & 0.0122 & 0.0921 & 0.0927 & 0.1319 & 0.1363 \\
\hline Med. bias & -0.0362 & -0.0390 & -0.0385 & 0.0173 & 0.0692 & 0.0494 & 0.1256 & 0.0449 \\
\hline RMSE & 0.1706 & 0.1801 & 0.1997 & 0.2042 & 0.3029 & 0.3086 & 0.4654 & 0.5302 \\
\hline MAD & 0.1350 & 0.1385 & 0.1580 & 0.1619 & 0.2295 & 0.2239 & 0.3540 & 0.3517 \\
\hline \multicolumn{9}{|l|}{$n=200$} \\
\hline Mean bias & -0.0236 & -0.0299 & 0.0165 & -0.0158 & 0.0746 & 0.0824 & 0.0823 & -0.0511 \\
\hline Med. bias & -0.0274 & -0.0344 & 0.0076 & -0.0439 & 0.0534 & 0.0530 & 0.1005 & 0.0264 \\
\hline RMSE & 0.1332 & 0.1391 & 0.1746 & 0.1670 & 0.2360 & 0.2521 & 0.3299 & 0.3604 \\
\hline MAD & 0.1051 & 0.1103 & 0.1344 & 0.1352 & 0.1786 & 0.1878 & 0.2530 & 0.2683 \\
\hline \multicolumn{9}{|l|}{$n=400$} \\
\hline Mean bias & -0.0339 & -0.0266 & -0.0188 & -0.0030 & 0.0507 & 0.0602 & 0.0512 & 0.0123 \\
\hline Med. bias & -0.0377 & -0.0269 & -0.0289 & 0.0037 & 0.0675 & 0.0319 & 0.0649 & -0.0410 \\
\hline RMSE & 0.1167 & 0.1072 & 0.1295 & 0.1324 & 0.2162 & 0.2275 & 0.2522 & 0.2992 \\
\hline MAD & 0.0908 & 0.0809 & 0.0996 & 0.1084 & 0.1657 & 0.1715 & 0.1978 & 0.2428 \\
\hline
\end{tabular}

Table 16

WNQN estimator: heteroskedastic design - normal errors

\begin{tabular}{|c|c|c|c|c|c|c|c|c|}
\hline & \multicolumn{2}{|c|}{$25 \%$ cens. } & \multicolumn{2}{|l|}{$40 \%$ cens. } & \multicolumn{2}{|l|}{$55 \%$ cens. } & \multicolumn{2}{|l|}{$65 \%$ cens. } \\
\hline & $\varepsilon=0.025$ & $\varepsilon=0.05$ & $\varepsilon=0.025$ & $\varepsilon=0.05$ & $\varepsilon=0.025$ & $\varepsilon=0.05$ & $\varepsilon=0.025$ & $\varepsilon=0.05$ \\
\hline \multicolumn{9}{|l|}{$n=100$} \\
\hline Mean bias & -0.0570 & -0.0448 & -0.0886 & -0.0489 & -0.0186 & -0.0026 & 0.2489 & 0.1677 \\
\hline Med. bias & -0.0564 & -0.0605 & -0.0786 & -0.0294 & 0.0133 & -0.0018 & 0.2154 & 0.1604 \\
\hline RMSE & 0.2241 & 0.1880 & 0.3500 & 0.2734 & 0.5835 & 0.4259 & 1.4587 & 1.0041 \\
\hline MAD & 0.1779 & 0.1494 & 0.2611 & 0.2090 & 0.4264 & 0.3189 & 0.9899 & 0.6858 \\
\hline \multicolumn{9}{|l|}{$n=200$} \\
\hline Mean bias & -0.0518 & -0.0600 & -0.0663 & -0.0900 & -0.0867 & -0.0725 & 0.1187 & 0.0428 \\
\hline Med. bias & -0.0562 & -0.0608 & -0.0528 & -0.0852 & -0.0832 & -0.0761 & 0.1182 & 0.0211 \\
\hline RMSE & 0.1545 & 0.1536 & 0.2380 & 0.1958 & 0.3726 & 0.2845 & 0.5704 & 0.4959 \\
\hline MAD & 0.1219 & 0.1224 & 0.1815 & 0.1575 & 0.2833 & 0.2177 & 0.4443 & 0.3795 \\
\hline \multicolumn{9}{|l|}{$n=400$} \\
\hline Mean bias & -0.0548 & -0.0549 & -0.1195 & -0.0957 & -0.0557 & -0.0556 & -0.0730 & -0.0419 \\
\hline Med. bias & -0.0529 & -0.0533 & -0.1144 & -0.1064 & -0.0556 & -0.0667 & -0.0717 & -0.0408 \\
\hline RMSE & 0.1207 & 0.1123 & 0.1934 & 0.1737 & 0.2760 & 0.2445 & 0.4653 & 0.3540 \\
\hline MAD & 0.0965 & 0.0882 & 0.1530 & 0.1432 & 0.2090 & 0.1881 & 0.3392 & 0.2703 \\
\hline
\end{tabular}


Table 17

WNQ estimator: heteroskedastic design - Laplace errors

\begin{tabular}{|c|c|c|c|c|c|c|c|c|}
\hline & \multicolumn{2}{|l|}{$25 \%$ cens. } & \multicolumn{2}{|l|}{$40 \%$ cens. } & \multicolumn{2}{|c|}{$55 \%$ cens. } & \multicolumn{2}{|l|}{$65 \%$ cens. } \\
\hline & $\varepsilon=0.025$ & $\varepsilon=0.05$ & $\varepsilon=0.025$ & $\varepsilon=0.05$ & $\varepsilon=0.025$ & $\varepsilon=0.05$ & $\varepsilon=0.025$ & $\varepsilon=0.05$ \\
\hline \multicolumn{9}{|l|}{$n=100$} \\
\hline Mean bias & 0.0035 & -0.0108 & 0.0478 & 0.0447 & 0.1130 & 0.1143 & 0.2061 & 0.1767 \\
\hline Med. bias & -0.0195 & -0.0300 & 0.0076 & 0.0071 & 0.0478 & 0.0731 & 0.0613 & 0.1373 \\
\hline RMSE & 0.2327 & 0.2062 & 0.2939 & 0.3025 & 0.4330 & 0.4239 & 0.8855 & 0.7343 \\
\hline MAD & 0.1827 & 0.1555 & 0.2213 & 0.2324 & 0.3225 & 0.3180 & 0.5754 & 0.5284 \\
\hline \multicolumn{9}{|l|}{$n=200$} \\
\hline Mean bias & -0.0114 & -0.0221 & 0.0355 & 0.0227 & 0.0927 & 0.0657 & 0.1592 & -0.0995 \\
\hline Med. bias & -0.0253 & -0.0352 & 0.0223 & 0.0150 & 0.0589 & 0.0304 & 0.1870 & -0.0997 \\
\hline RMSE & 0.1617 & 0.1430 & 0.2037 & 0.2129 & 0.2976 & 0.3192 & 0.4543 & 0.4645 \\
\hline MAD & 0.1237 & 0.1142 & 0.1618 & 0.1617 & 0.2162 & 0.2342 & 0.3408 & 0.3488 \\
\hline \multicolumn{9}{|l|}{$n=400$} \\
\hline Mean bias & -0.0303 & -0.0285 & 0.0048 & -0.0116 & 0.0682 & 0.0622 & 0.0224 & -0.0911 \\
\hline Med. bias & -0.0378 & -0.0398 & -0.0046 & -0.0210 & 0.0440 & 0.0215 & 0.0965 & -0.0643 \\
\hline RMSE & 0.1180 & 0.1146 & 0.1762 & 0.1728 & 0.2575 & 0.2660 & 0.4197 & 0.4290 \\
\hline MAD & 0.0905 & 0.0917 & 0.1354 & 0.1313 & 0.1896 & 0.1963 & 0.3282 & 0.3096 \\
\hline
\end{tabular}

Table 18

WNQN estimator: heteroskedastic design - Laplace errors

\begin{tabular}{|c|c|c|c|c|c|c|c|c|}
\hline & \multicolumn{2}{|l|}{$25 \%$ cens. } & \multicolumn{2}{|l|}{$40 \%$ cens. } & \multicolumn{2}{|l|}{$55 \%$ cens. } & \multicolumn{2}{|l|}{$65 \%$ cens. } \\
\hline & $\varepsilon=0.025$ & $\varepsilon=0.05$ & $\varepsilon=0.025$ & $\varepsilon=0.05$ & $\varepsilon=0.025$ & $\varepsilon=0.05$ & $\varepsilon=0.025$ & $\varepsilon=0.05$ \\
\hline \multicolumn{9}{|l|}{$n=100$} \\
\hline Mean bias & -0.0433 & -0.0476 & -0.0298 & -0.0587 & -0.0830 & -0.0928 & 0.0961 & 0.2657 \\
\hline Med. bias & -0.0479 & -0.0435 & 0.0208 & -0.0790 & -0.0546 & -0.0948 & 0.2610 & 0.2244 \\
\hline RMSE & 0.2546 & 0.2065 & 0.4854 & 0.3606 & 0.8465 & 0.6767 & 1.8300 & 1.2762 \\
\hline MAD & 0.2016 & 0.1604 & 0.3357 & 0.2735 & 0.6038 & 0.4886 & 1.3019 & 0.8157 \\
\hline \multicolumn{9}{|l|}{$n=200$} \\
\hline Mean bias & -0.0601 & -0.0592 & -0.0839 & -0.0569 & -0.0552 & -0.0872 & -0.0761 & 0.1391 \\
\hline Med. bias & -0.0553 & -0.0617 & -0.0930 & -0.0595 & -0.0014 & -0.1108 & -0.0245 & 0.1150 \\
\hline RMSE & 0.1667 & 0.1523 & 0.3048 & 0.2328 & 0.5066 & 0.4410 & 0.9427 & 0.6826 \\
\hline MAD & 0.1271 & 0.1202 & 0.2316 & 0.1842 & 0.3943 & 0.3230 & 0.6320 & 0.4993 \\
\hline \multicolumn{9}{|l|}{$n=400$} \\
\hline Mean bias & -0.0531 & -0.0421 & -0.0840 & -0.0852 & -0.0415 & -0.0625 & -0.0367 & -0.0953 \\
\hline Med. bias & -0.0577 & -0.0432 & -0.0926 & -0.0837 & -0.0115 & -0.0120 & -0.0386 & -0.0604 \\
\hline RMSE & 0.1278 & 0.1140 & 0.2227 & 0.1826 & 0.3262 & 0.3090 & 0.5826 & 0.4644 \\
\hline MAD & 0.1006 & 0.0920 & 0.1714 & 0.1427 & 0.2624 & 0.2456 & 0.4303 & 0.3501 \\
\hline
\end{tabular}


For each of these restrictions on the error terms, we simulated for four different values of the intercept term $a$, corresponding to censoring levels of $25 \%, 40 \%, 55 \%$ and $65 \%$. Each design was replicated 401 times, for sample sizes of 100, 200 and 400. The tables report mean bias, median bias, root mean squared error, and mean absolute deviation for all the estimators.

Tables 1-4 report results for four maximum likelihood estimators based on the assumption of normal errors. The first estimator, referred to in the tables as TOB1, is the classical Tobit estimator, which assumes homoskedasticity and simultaneously estimates the scale factor along with the intercept and slope coefficient. The second estimator, referred to as TOB2, allows for heteroskedasticity, and correctly specifies the scale function as $\mathscr{C} \mathrm{e}^{0.4 x_{i}^{2}}$. To illustrate the sensitivity of MLE to specification of the scale function, results for MLE with misspecified scale functions are reported in Tables 5 and 6. These estimators, referred to as TOB3 and TOB4, correspond to scale function specifications of $\left|x_{i}\right|$ and $\mathrm{e}^{\left|x_{i}\right|}$, respectively.

Tables 7-10 report results for Powell's quantile regression estimator, using the quantiles $\alpha=0.5$ and 0.75 . Tables $11-18$ report the results for the two estimators introduced in this paper.

Our simulation study was performed in GAUSS. The ML estimators were computed using the BFGS descent algorithm. To compute the CLAD estimator, we adopted the iterative linear programming method introduced in Buchinsky (1994).$^{10}$ The first stage of our two estimators was computed using the linear programming method discussed in Buchinsky (1994), which is guaranteed to converge to the global minimum. This method was used to fit a local cubic model for the conditional quantile functions.

Implementation of the two new estimators required selection of the weighting function, the bandwidth, and the quantile pair. Following Buchinsky and Hahn (1998) we adopted the weighting function

$$
\begin{aligned}
\omega\left(q_{\alpha}\right)= & \left(\frac{\mathrm{e}^{q_{\alpha}-2 c}}{1+\mathrm{e}^{q_{\alpha}-2 c}}-\frac{\mathrm{e}^{-c}}{1+\mathrm{e}^{-c}}\right)\left(\frac{2+\mathrm{e}^{c}+\mathrm{e}^{-c}}{\mathrm{e}^{c}-\mathrm{e}^{-c}}\right) I\left[c<q_{\alpha}<3 c\right] \\
& +I\left[q_{\alpha}>3 c\right]
\end{aligned}
$$

and set $c=0.05$. For the bandwidth, we set the rate to $n^{-2 / 7}$. With $p=2$ and $d=1$, this rate satisfies Assumption BC. The constant was selected by a weak grid search over the interval $[1,4]$ and the value which minimized the sum of square residuals was chosen.

\footnotetext{
${ }^{10}$ This algorithm only guarantees convergence to a local minimum of the objective function. However, existing results, such as those found in Buchinsky (1994) indicate that this algorithm works well in practice. In preliminary trials, we used LAD, OLS, and the true parameter values as starting values and results were quite insensitive to the choice of starting value in the designs considered. The tables report results for the OLS starting value.
} 
The selection of the quantile pair in finite samples is governed by two factors. The first is that for the full rank condition to be satisfied in finite samples, it is clearly necessary that both quantiles be significantly larger than the level of censoring in the data. However, precision of the estimators is also sacrificed if the quantiles get arbitrarily close to one, as the density of the residuals becomes very small. These were the factors mentioned in Powell (1986a) in selecting the appropriate quantile for the homoskedastic model. Optimally weighing in both considerations is a difficult problem as it involves knowledge of the regressor distribution, the parameter $\beta_{0}$, the scale function, and the density of the homoskedastic component of the error term. While a multiple stage approach (involving a preliminary quantile pair that can be used to estimate each of the unknown values in the first stage) may be plausible, we leave its development and asymptotic properties for future work. Here we only propose a simple rule of thumb approach which is only based on the level of censoring in the data. Let $p_{\mathrm{c}}$ denote the fraction of observations censored in the data set. We propose setting $\alpha_{1}$ to $\left(1+p_{\mathrm{c}}\right) / 2+\varepsilon$ and $\alpha_{2}$ to $\left(3+p_{\mathrm{c}}\right) / 4-\varepsilon$ where $\varepsilon$ is a small number, so the distance between quantile values is between 0.05 and 0.10 . This picks quantiles in a neighborhood of the midpoint of the interval $\left[p_{\mathrm{c}}, 1\right]$, and crudely takes into account each of these factors. For this simulation study, results are reported for $\varepsilon=0.025$ and 0.050 (denoted in the tables by superscripts) to explore sensitivity to the quantile pair choice.

Tables 1-6 illustrate the advantages and disadvantages of adopting a parametric estimation approach. When the model is correctly specified, as is the case for TOB1 in design 1, MLE yields estimators which are very precise at even $n=100$. TOB 1 performs quite well in design 2 and TOB 2 performs well in all designs, even though the error distribution is sometimes misspecified, confirming that distributional misspecification is not as serious a problem as heteroskedasticity. Tables 3-6 illustrate the main disadvantage of the likelihood approach. Specifically, when conditional heteroskedasticity is parametrically misspecified, the estimator breaks down, as illustrated by the poor performance of TOB1, TOB3 and TOB4 under designs 3 and 4.

The results of Tables 7-10 agree with the previous discussion regarding the quantile regression estimator that motivated the estimators introduced in this paper. For censoring levels less than $50 \%$ both quantile regression estimators perform very well in homoskedastic designs when $\alpha \leqslant 0.5$. However, for censoring levels at $65 \%$, only the quantile estimator with $\alpha=0.75$ performed well. The CLAD estimator clearly fails in this case, with RMSEs and biases not shrinking with the sample size. For heteroskedastic designs, however, only the CLAD estimator, corresponding to $\alpha=0.5$ performs well at the lower censoring levels. The quantile estimator with $\alpha=0.75$, which is inconsistent in the heteroskedastic designs, performs very poorly, even for censoring levels as low as $40 \%$. The CLAD performs even worse than in the homoskedastic design when the censoring is $65 \%$ and the errors are Laplace. 
On the other hand, Tables 11-18 illustrate that the WNQN and WNQ estimators performed quite well for all degrees of censoring. ${ }^{11}$ Though the WNQN estimator exhibits somewhat large RMSEs in the heteroskedastic designs for sample sizes of 100 , the estimators clearly exhibit consistency, and except for the infeasible TOB2 procedure, outperform the other estimators for the heteroskedastic designs when the degree of censoring is heavy. Also, neither estimator was very sensitive to the choice of quantile pair.

As expected, the WNQ performed better than the WNQN in the Laplace designs, and somewhat surprisingly, had a smaller RMSE for the normal designs. We have a possible explanation for this result. The finite sample performances of both estimators rely heavily on the performance of the first step nonparametric quantile estimates. For WNQ, whose formulation is based on Eq. (2.8), there may be a certain offsetting effect on the imprecision caused by the first step nonparametric estimation, as the quantile estimates are used as both dependent and independent variables. On the other hand, there is no such offsetting effect for WNQN.

In summary, the results of our simulation study regarding the CLAD follow the predictions of the theory behind it, and agree with certain empirical problems which have been encountered in practice. Furthermore, the positive results for the two estimators we introduce here suggest they are viable alternatives to using the CLAD. We conclude this section with two additional remarks regarding our simulation results. First, it should be noted that for all designs we considered, there is a fraction of observations for which the index $x_{i}^{\prime} \beta_{0}$ is positive. Thus it is not necessary for the full rank condition to be literally violated for the CLAD estimator to break down in finite samples. Second, it should be noted that the designs considered only involved one regressor. It is expected that the results for the WNQN and WNQ would be worse if the number of regressors were increased, as the first stage estimator would suffer from the usual curse of dimensionality, and possibly have a (second-order) effect on the second stage estimator. However, as alluded to in the previous section, it would also be possible to improve upon our results by considering several quantile pairs, and combining the resulting estimators in some optimal way.

\section{Summary and concluding remarks}

This paper introduces two new estimators for censored regression models with conditional heteroskedasticity of very general forms. One of the estimators

\footnotetext{
${ }^{11}$ As alluded to earlier, the limiting covariance matrix of the WNQ estimator will be singular in the first two designs. It is mentioned in footnote 9 that estimability of the slope parameter is not an issue, and the results in Tables 11 and 13 confirm that it can be estimated precisely despite the near collinearity in the second stage regression.
} 
requires no parametric specification of the homoskedastic error term which is multiplied by a scale function. The advantage of these estimators is that they allow for more general full rank conditions under heteroskedasticity than estimators based on a conditional median restriction, notably Powell's CLAD estimator. This generalization proves especially useful in the presence of heavily censored data.

The proposed estimators are shown to have desirable asymptotic properties, and a simulation study indicates that the estimators have favorable finite sample properties at various degrees of censoring. The work here leaves open many important questions, suggesting areas for future research. First, we hope to develop data-driven methods to select the quantile pair. A separate but related issue we leave for future work is the possibility of linearly combining various estimators of $\beta_{0}$ based on different quantile pairs to construct a more efficient estimator. For a finite set of quantile pairs, the expressions for $\delta\left(y_{i}, x_{i}\right)$, $\delta^{*}\left(y_{i}, x_{i}, z_{i}\right)$ can be used to easily derive the limiting covariance matrix of the vector of estimators, which can be used to construct the optimal weighting matrix in a fashion analogous to Powell (1986a). Also of interest would be the difficult problem of determining whether a fine enough grid of quantiles can be used to approach the semiparametric efficiency bound, which itself has yet to be derived for the censored regression model under multiplicative heteroskedasticity.

Furthermore, the estimators here suggest new testing procedures as well. A test for normality could be constructed by comparing the two estimators, and a Hausman-type test for heteroskedasticity could be constructed by comparing the second estimator to one of several estimators in the literature based on the independence restriction.

We also mention that the procedures introduced in this paper can be extended to other models, suggesting other areas for further work. For example, Powell's (1986b) symmetrically trimmed estimators also require a positive index for identification, which might be able to be relaxed in a similar fashion. Also, estimators which are bivariate extensions of these symmetrically trimmed estimators (e.g. Honoré, 1992; Honoré and Powell, 1994; Honoré et al., 1997) have analogous rank conditions which possibly can be relaxed by adopting similar procedures.

\section{Acknowledgements}

We are grateful to B.E. Honoré, J.L. Powell and two anonymous referees for their helpful comments.

\section{Appendix A}

To keep expressions notationally simple, in this section we let $q_{1 i}$ and $q_{2 i}$ denote $q_{\alpha_{1}}\left(x_{i}\right)$ and $q_{\alpha_{2}}\left(x_{i}\right)$, respectively. We denote estimated values by 
$\hat{q}_{1 i}, \hat{q}_{2 i}$. We let $\tau_{i}, \omega_{i}, \hat{\omega}_{i}$ denote $\tau\left(x_{i}\right), \omega\left(q_{\alpha_{1}}\left(x_{i}\right)\right), \omega\left(\hat{q}_{\alpha_{1}}\left(x_{i}\right)\right)$, respectively. Also, we let $C_{n i}$ denote $C_{n}\left(x_{i}\right)$, and let $N_{n}\left(x_{i}\right)=\sum_{j \neq i} I\left[x_{j} \in C_{n i}\right]$. Finally, for any matrix $A$ with elements $a_{i j}$, we let $\|A\| \operatorname{denote}\left(\sum_{i, j} a_{i j}^{2}\right)^{1 / 2}$.

\section{A.1. Proof of Theorem 1}

We concentrate exclusively on deriving the limiting distribution for the first estimator, as very similar arguments can be used for the second estimator. Here, we let $\Delta_{21} q_{i}$ and $\Delta_{21} \hat{q}_{i}$ denote $\left(c_{\alpha_{2}}^{Z} q_{\alpha_{1}}\left(x_{i}\right)-c_{\alpha_{1}}^{Z} q_{\alpha_{2}}\left(x_{i}\right)\right) /\left(c_{\alpha_{2}}^{Z}-c_{\alpha_{1}}^{Z}\right)$ and $\left(c_{\alpha_{2}}^{Z} \hat{q}_{\alpha_{1}}\left(x_{i}\right)-c_{\alpha_{1}}^{Z} \hat{q}_{\alpha_{2}}\left(x_{i}\right)\right) /\left(c_{\alpha_{2}}^{Z}-c_{\alpha_{1}}^{Z}\right)$, respectively. The derivation involves working with the relationship

$$
\begin{aligned}
\widehat{\beta}-\beta_{0} & =\left(\frac{1}{2} \sum_{i=1}^{n} \tau_{i} \hat{\omega}_{i} x_{i} x_{i}^{\prime}\right)^{-1}\left(\frac{1}{2} \sum_{i=1}^{n} \tau_{i} \hat{\omega}_{i} x_{i}\left(\Delta_{21} \hat{q}_{i}-x_{i}^{\prime} \beta_{0}\right)\right) \\
& \equiv \hat{J}^{-1} \hat{S} .
\end{aligned}
$$

We aim to derive the probability limit of the $\hat{J}$ of the above expression and a linear representation for the $\hat{S}$. We begin by establishing the following two lemmas, which correspond to two uniform convergence results for the nonparametric estimator of the conditional quantile function. They are stated for $\hat{q}_{1 i}$ but apply to $\hat{q}_{2 i}$ as well. The first lemma establishes a rate uniform over points where the quantile function is bounded away from the censoring point. The result follows directly from the uniform rates derived in Chaudhuri (1991b) and Chaudhuri et al. (1997).

Lemma 1 (Chaudhuri et al., 1997, Lemma 4.3a).

$$
\max _{1 \leqslant i \leqslant n, q_{1 i} \geqslant c / 2} \tau_{i}\left|\hat{q}_{1 i}-q_{1 i}\right|=o_{p}\left(n^{-1 / 4}\right) \text {. }
$$

The second uniform result involves an exponential bound for points in a neighborhood of the censoring point:

Lemma 2. Let $\mathscr{X}_{\mathrm{c}}$ denote the set

$$
\left\{x_{i} \in \mathscr{X}, q_{1 i} \leqslant c / 2\right\}
$$

and let $A_{n}$ denote the event

$$
\left\{\hat{q}_{1 i} \geqslant c \text { for all } x_{i} \in \mathscr{X}_{\mathrm{c}}\right\}
$$

then there exist constants $C_{1}, C_{2}$ such that

$$
P\left(A_{n}\right) \leqslant C_{1} \mathrm{e}^{-C_{2} n h_{n}^{d_{c}}}
$$

Proof. We first derive a similar result for the nonparametric estimator which fits a polynomial of degree 0 , and denote this by $\hat{q}_{1 i}^{0}$. We consider attaining an 
exponential bound for the probability of the event

$$
\bar{A}_{n}=\left\{\hat{q}_{1 i}^{0} \geqslant 3 c / 4 \text { for all } x_{i} \in \mathscr{X}_{\mathrm{c}}\right\} .
$$

For a pair of positive constants $c_{1}<c_{2}$ we define the event $E_{n}$ as

$$
\left\{c_{1} n h_{n}^{d_{\mathrm{c}}} \leqslant N_{n}\left(x_{i}\right) \leqslant c_{2} n h_{n}^{d_{\mathrm{c}}} \text { for all } x_{i} \in \mathscr{X}_{\mathrm{c}}\right\} .
$$

By Theorem 3.1(i) in Chaudhuri (1991b), we can choose $c_{1}, c_{2}$ such that

$$
\mathrm{P}\left(E_{n}^{\mathrm{c}}\right) \leqslant c_{3} \mathrm{e}^{-c_{4} n h_{n}^{d_{c}}}
$$

where $c_{3}, c_{4}$ are positive constants, and $E_{n}^{\mathrm{c}}$ denotes the complement of the event $E_{n}$. Thus it will suffice to derive an exponential rate for the probability of the event $\bar{A}_{n} \cap E_{n}$. We note that $\bar{A}_{n}$ implies the event

$$
\frac{1}{N_{n}\left(x_{i}\right)} \sum_{x_{j} \in C_{n i}} I\left[y_{j} \geqslant 3 c / 4\right] \geqslant\left(1-\alpha_{1}\right) \text { for all } x_{i} \in \mathscr{X}_{\mathrm{c}} .
$$

Now, by the continuity of $q_{1 i}$ and the compactness of $\mathscr{X}$, we have for $n$ larger than some $N_{0}, q_{1 j}<2 c / 3$ if $x_{j} \in C_{n i}$ for all $x_{i} \in \mathscr{X}_{\mathrm{c}}$.

Thus by Assumption ED there is a positive constant $\lambda_{1}$ such that for $x_{j} \in C_{n i}$

$$
\mathrm{P}\left(y_{j} \geqslant 3 c / 4 \mid x_{j}\right) \leqslant \mathrm{P}\left(v_{1 j} \geqslant c / 12 \mid x_{j}\right) \leqslant\left(1-\alpha_{1}\right)-\lambda_{1}
$$

and the probability of the event $\bar{A}_{n} \cap E_{n}$ is bounded above by

$$
\begin{aligned}
& \mathrm{P}\left(\sum_{x_{j} \in C_{n i}} I\left[y_{j} \geqslant 3 c / 4\right]-E\left[I\left[y_{j} \geqslant 3 c / 4\right] \mid x_{j}\right] \geqslant c_{1} n h^{d_{\mathrm{c}}} \lambda_{1} \cap E_{n}\right) \\
& \leqslant \mathrm{e}^{-2 \lambda_{1}^{2} c_{1}^{2} c_{2}^{-1} n h_{n}^{d_{c}}},
\end{aligned}
$$

where the exponential bound follows by Hoeffding's inequality. Thus the exponential bound follows for $\hat{q}_{1 i}^{0}$ by picking $C_{1}$ and $C_{2}$ such that the bounds in (A.2) and (A.4) are satisfied. Finally, the conclusion of the lemma follows for $\hat{q}_{1 i}$ by showing that

$$
\left|\hat{q}_{1 i}-\hat{q}_{1 i}^{0}\right|<c / 4 \text { for all } x_{i} \in \mathscr{X} .
$$

This result becomes apparent by expressing the local polynomial estimator $\hat{q}_{1 i}$ as an estimator with polynomial of degree 0 and dependent variable equal to

$$
y_{j}^{*}=y_{j}-\sum_{l=1}^{s(A)} \hat{\theta}_{(1)}\left(x_{j}^{(\mathrm{c})}-x_{i}^{(\mathrm{c})}\right)^{b_{l}}
$$


where $\hat{\theta}_{(1)}$ (which are constrained to lie in a compact set by construction) are the local polynomial estimators of the quantile function and its derivatives. Thus $\left|y_{j}^{*}-y_{j}\right|<c / 4$ for all $x_{i} \in \mathscr{X}$ for all $n$ larger than some $N_{1}$, as the local polynomial estimators are restricted to lie in a bounded set. This establishes (A.5).

These uniform results enable us to immediately derive the probability limit of $\hat{J}$ :

Lemma 3.

$$
\left(\frac{1}{n} \sum_{i=1}^{n} \tau_{i} \hat{\omega}_{i} x_{i} x_{i}^{\prime}\right) \stackrel{p}{\rightarrow} J .
$$

Proof. A mean value expansion of $\hat{\omega}_{i}$ around $\omega_{i}$ yields

$$
\frac{1}{n} \sum_{i=1}^{n} \tau_{i} \omega_{i} x_{i} x_{i}^{\prime}+\frac{1}{n} \sum_{i=1}^{n} \tau_{i} \omega_{i}^{*}\left(\hat{q}_{1 i}-q_{1 i}\right) x_{i} x_{i}^{\prime},
$$

where $\omega_{i}^{*}$ denotes the weighting function evaluated at an intermediate value. By a WLLN the first term in the above expression converges in probability to $J$. We decompose the second term as

$$
\begin{aligned}
& \frac{1}{n} \sum_{i=1}^{n} \tau_{i} \omega_{i}^{*}\left(\hat{q}_{1 i}-q_{1 i}\right) x_{i} x_{i}^{\prime} I\left[q_{1 i} \geqslant c / 2\right] \\
& +\frac{1}{n} \sum_{i=1}^{n} \tau_{i} \omega_{i}^{*}\left(\hat{q}_{1 i}-q_{1 i}\right) x_{i} x_{i}^{\prime} I\left[q_{1 i}<c / 2\right] .
\end{aligned}
$$

By Lemma 1 the first summation is $o_{p}(1)$. Note for the second summation each nonzero term in the summation satisfies $\hat{q}_{1 i} \geqslant c$ and $q_{1 i}<c / 2$ so we can bound this summation by a term which is $o_{p}(1)$ by Lemma 2 .

We next establish a linear representation for $\hat{S}$ in (A.1). We decompose this term into

$$
\frac{1}{n} \sum_{i=1}^{n} \tau_{i} \hat{\omega}_{i} x_{i}\left(\Delta_{21} \hat{q}_{i}-\Delta_{21} q_{i}\right)+\frac{1}{n} \sum_{i=1}^{n} \tau_{i} \hat{\omega}_{i} x_{i}\left(\Delta_{21} q_{i}-x_{i}^{\prime} \beta_{0}\right) .
$$

The following lemma establishes the asymptotic neglibility of the second term in the decomposition:

Lemma 4.

$$
\frac{1}{n} \sum_{i=1}^{n} \tau_{i} \hat{\omega}_{i} x_{i}\left(\Delta_{21} q_{i}-x_{i}^{\prime} \beta_{0}\right)=\mathrm{o}_{\mathrm{p}}\left(n^{-1 / 2}\right) .
$$


Proof. A mean value expansion around $\omega_{i}$ implies that we are left with

$$
\frac{1}{n} \sum_{i=1}^{n} \tau_{i} \omega_{i}^{*} x_{i}\left(\hat{q}_{1 i}-q_{1 i}\right)\left(\Delta_{21} q_{i}-x_{i}^{\prime} \beta_{0}\right) I\left[q_{1 i}<c / 2\right]
$$

which is $o_{p}\left(n^{-1 / 2}\right)$ by Lemma 2 .

We next decompose the first term in (A.9) as

$$
\frac{1}{n} \sum_{i=1}^{n} \tau_{i} \hat{\omega}_{i} x_{i} \dot{c}_{1}\left(\hat{q}_{1 i}-q_{1 i}\right)-\frac{1}{n} \sum_{i=1}^{n} \tau_{i} \hat{\omega}_{i} x_{i} \dot{c}_{2}\left(\hat{q}_{2 i}-q_{2 i}\right),
$$

where $\dot{c}_{1}=c_{\alpha_{2}}^{Z} /\left(c_{\alpha_{2}}^{Z}-c_{\alpha_{1}}^{Z}\right)$ and $\dot{c}_{2}=c_{\alpha_{1}}^{Z} /\left(c_{\alpha_{2}}^{Z}-c_{\alpha_{1}}^{Z}\right)$. The following lemma represents the first term in (A.11) as a sum of i.i.d. mean 0 vectors:

Lemma 5.

$$
\frac{1}{n} \sum_{i=1}^{n} \tau_{i} \hat{\omega}_{i} x_{i} \dot{c}_{1}\left(\hat{q}_{1 i}-q_{1 i}\right)=\frac{1}{n} \sum_{i=1}^{n} \delta_{\alpha_{1}}\left(y_{i}, x_{i}\right)+\mathrm{o}_{\mathrm{p}}\left(n^{-1 / 2}\right) .
$$

Proof. By the same mean value expansion, we can replace $\hat{\omega}_{i}$ with $\omega_{i}$ in the above expression. The remainder term is $o_{p}\left(n^{-1 / 2}\right)$ by Lemmas 1 and 2 . The next step is to 'plug in' a linear representation for $\left(\hat{q}_{1 i}-q_{1 i}\right)$. Local Bahadur representations for the local polynomial quantile regression estimator have been established in Chaudhuri (1991a) and Chaudhuri et al. (1997). Following identical steps as taken in Chaudhuri et al. (1997) (see also Chen and Khan, 1999), we are left with

$$
\begin{aligned}
& \frac{1}{n(n-1)} \sum_{i \neq j} \tau_{i} \omega_{i} x_{i} f_{v_{1}, X}\left(0, x_{i}\right)^{-1} h_{n}^{-d_{c}}\left(\alpha_{1}-I\left[y_{j} \leqslant q_{1 j}\right]\right) I\left[x_{j} \in C_{n i}\right] \\
& +\mathrm{o}_{\mathrm{p}}\left(n^{-1 / 2}\right),
\end{aligned}
$$

where $f_{v_{1}, X}$ denotes the joint density function of $v_{1 i}, x_{i}$. Let $\xi_{i}=\left(y_{i}, x_{i}^{\prime}\right)^{\prime}$, and let $\mathscr{F}_{n}\left(\xi_{i}, \xi_{j}\right)$ denote the expression in the above double summation. We wish to establish

$$
\frac{1}{n(n-1)} \sum_{i \neq j} \mathscr{F}_{n}\left(\xi_{i}, \xi_{j}\right)=\frac{1}{n} \sum_{i=1}^{n} \delta_{\alpha_{1}}\left(\xi_{i}\right)+\mathrm{o}_{\mathrm{p}}\left(n^{-1 / 2}\right)
$$

To do so, we first note that by a change of variables,

$$
\mathrm{E}\left[\left\|\mathscr{F}_{n}\left(\xi_{i}, \xi_{j}\right)\right\|^{2}\right]=\mathrm{O}\left(h_{n}^{-d_{c}}\right)=\mathrm{o}(n),
$$

where the second equality follows from Assumption BC. Let $\mathrm{E}\left[\mathscr{F}_{n}\left(\xi_{i}, \cdot\right)\right]$ and E $\left[\mathscr{F}_{n}\left(\cdot, \xi_{i}\right)\right]$ denote the expectation of $\mathscr{F}_{n}$ conditional on its first and second arguments, respectively. By Lemma 3.1 of Powell et al. (1989), noting that 
$\mathrm{E}\left[\mathscr{F}_{n}\left(\xi_{i}, \xi_{j}\right)\right]=\mathrm{E}\left[\mathscr{F}_{n}\left(\xi_{i}, \cdot\right)\right]=0$, we have

$$
\frac{1}{n(n-1)} \sum_{i \neq j} \mathscr{F}_{n}\left(\xi_{i}, \xi_{j}\right)=\frac{1}{n} \sum_{i=1}^{n} \mathrm{E}\left[\mathscr{F}_{n}\left(\cdot, \xi_{i}\right)\right]+\mathrm{o}_{\mathrm{p}}\left(n^{-1 / 2}\right) .
$$

Therefore, Eq. (A.14) will follow if it can be shown that

$$
\frac{1}{n} \sum_{i=1}^{n} \mathrm{E}\left[\mathscr{F}_{n}\left(\cdot, \xi_{i}\right)\right]=\frac{1}{n} \sum_{i=1}^{n} \delta_{\alpha_{1}}\left(\xi_{i}\right)+\mathrm{o}_{\mathrm{p}}\left(n^{-1 / 2}\right) .
$$

By a change of variables we can write $\mathrm{E}\left[\mathscr{F}_{n}\left(\cdot, z_{i}\right)\right]$ as

$$
\begin{aligned}
& \left(\alpha_{1}-I\left[y_{i} \leqslant q_{1 i}\right]\right) \\
& \quad \int_{[-1,1]^{d_{c}}} \tau\left(x_{i}^{(\mathrm{ds})}, x_{i}^{(\mathrm{c})}-t h_{n}\right) \omega\left(q_{\alpha_{1}}\left(x_{i}^{(\mathrm{ds})}, x_{i}^{(\mathrm{c})}-t h_{n}\right)\left(x_{i}^{(\mathrm{ds})}, x_{i}^{(\mathrm{c})}-t h_{n}\right)\right. \\
& f_{v_{1} \mid X}\left(0 \mid x_{i}^{(\mathrm{ds})}, x_{i}^{(\mathrm{c})}-t h_{n}\right)^{-1} \mathrm{~d} t
\end{aligned}
$$

which by the dominated convergence theorem converges almost surely to $\left(\alpha_{1}-I\left[y_{i} \leqslant q_{1 i}\right]\right) \tau_{i} \omega_{i} x_{i} f_{v_{1} \mid X}\left(0 \mid x_{i}\right)^{-1}$. Thus by another application of the dominated convergence theorem, we have $\mathrm{E}\left[\| \mathrm{E}\left[\mathscr{\mathscr { F }}_{n}\left(\cdot, \xi_{i}\right)-\delta_{1}\left(\xi_{i}\right) \|^{2}\right] \rightarrow 0\right.$. Therefore, (A.15) follows from Chebyshev's inequality, completing the proof.

By identical arguments, the following result can be established:

\section{Lemma 6.}

$$
\frac{1}{n} \sum_{i=1}^{n} \tau_{i} \hat{\omega}_{i} x_{i} \dot{c}_{2}\left(\hat{q}_{2 i}-q_{2 i}\right)=\frac{1}{n} \sum_{i=1}^{n} \delta_{\alpha_{2}}\left(y_{i}, x_{i}\right)+\mathrm{o}_{\mathrm{p}}\left(n^{-1 / 2}\right) .
$$

Combining Lemmas 5 and 6 completes the linear representation for $\hat{S}$ in (A.1). Combining this with Lemma 3 and applying Slutsky's theorem proves the theorem.

\section{A.2. Proof of Theorem 2}

Again, we only establish the proof for the components of the limiting covariance matrix for the estimator under normality. Here we let $\sigma_{i}$ and $\hat{\sigma}_{i}$ denote the scale function evaluated at $x_{i}$ and its estimate, respectively.

It follows immediately from Lemma 3 that $\widehat{J} \stackrel{p}{\rightarrow} J$. We establish the consistency of $\hat{\Omega}$. Let $\mathscr{V}_{\alpha_{j}, i}, j=1,2$, be defined by

$$
\left(\frac{\bar{c}^{Z}}{\Delta c^{Z}}+\frac{(-1)^{j}}{2}\right) \tau\left(x_{i}\right) \omega\left(q_{\alpha_{1}}\left(x_{i}\right)\right) \sigma_{i} \phi_{Z}\left(c_{\alpha_{j}}^{Z}\right)^{-1}
$$


and note that

$$
\begin{aligned}
\Omega= & \mathrm{E}\left[\left(\mathscr{V}_{\alpha_{2}, i}^{2}\left(\alpha_{2}\left(1-\alpha_{2}\right)\right)+\mathscr{V}_{\alpha_{1}, i}^{2}\left(\alpha_{1}\left(1-\alpha_{1}\right)\right)\right.\right. \\
& \left.\left.-2 \mathscr{V}_{\alpha_{2}, i} \mathscr{V}_{\alpha_{1}, i}\left(\alpha_{1}\left(1-\alpha_{2}\right)\right)\right) x_{i} x_{i}^{\prime}\right] .
\end{aligned}
$$

By the Cauchy Schwarz and triangle inequalities, and the law of large numbers, it will suffice to show (see, for example, Lemma 8.3 of Newey and McFadden, 1994) the following:

$$
\frac{1}{n} \sum_{i=1}^{n}\left|\widehat{\mathscr{V}}_{\alpha_{j}}\left(x_{i}\right)-\mathscr{V}_{\alpha_{j}}\left(y_{i}, x_{i}\right)\right|^{2}=\mathrm{o}_{\mathrm{p}}(1) \quad \text { for } j=1,2 .
$$

We show that (A.17) holds for $\alpha_{1}$. The same argument can be used for $\alpha_{2}$. We note by Lemmas 1 and 2, and that $\mathscr{X}$ is a bounded set, it will suffice to show

$$
\frac{1}{n_{i}} \sum_{i=1}^{n} \omega_{i} \tau_{i}\left(\hat{\sigma}_{i}-\sigma_{i}\right)^{2}=\mathrm{o}_{\mathrm{p}}(1)
$$

To show (A.18), we note that $\left(\hat{\sigma}_{i}-\sigma_{i}\right)^{2}$ is proportional to a term bounded above by

$$
\tau_{i} \omega_{i}\left(\left\|x_{i}\right\|^{2}\left\|\hat{\beta}-\beta_{0}\right\|^{2}+\left|\hat{q}_{1 i}-q_{1 i}\right|^{2}\right),
$$

so (A.18) follows from Lemma 1 and Theorem 1.

\section{References}

Amemiya, T., 1985. Advanced Econometrics. Harvard University Press, Cambridge, MA.

Andrews, D.W.K., 1994. Empirical process methods in econometrics. In: Engle, R.F., McFadden, D. (Eds.), Handbook of Econometrics, Vol. 4. North-Holland, Amsterdam.

Buchinsky, M., 1994. Changes in the U.S. wage structure 1963-1987: application of quantile regression. Econometrica 62, 405-458.

Buchinsky, M., Hahn, J., 1998. An alternative estimator for the censored quantile regression model. Econometrica 66, 653-672.

Chaudhuri, P., 1991a. Nonparametric quantile regression. Annals of Statistics 19, 760-777.

Chaudhuri, P., 1991b. Global nonparametric estimation of conditional quantiles and their derivatives. Journal of Multivariate Analysis 39, 246-269.

Chaudhuri, P., Doksum, K., Samarov, A., 1997. On average derivative quantile regression. Annals of Statistics 25, 715-744.

Chay, K.Y., 1995. Evaluating the impact of the 1964 civil rights act on the economic status of black men using censored longitudinal earnings data. Manuscript, Princeton University, unpublished.

Chen, S., Khan, S., 1999. Semiparametric estimation of a partially linear censored regression model. Forthcoming, Econometric Theory.

Donald, S.G., 1995. Two-step estimation of heteroscedastic sample selection models. Journal of Econometrics 65, 347-380.

Engle, R.F., 1982. Autoregressive conditional heteroskedasticity with estimates of the variance of United Kingdom inflations. Econometrica 50, 987-1008.

Fan, J., Gijbels, I., 1996. Local Polynomial Modelling and its Applications. Chapman \& Hall, New York. 
Harvey, A.C., 1976. Estimating regression models with multiplicative heteroskedasticity. Econometrica $44,461-465$.

Honoré, B.E., 1992. Trimmed LAD and least squares estimation of truncated and censored regression models with fixed effects. Econometrica 60, 533-565.

Honoré, B.E., Kyriazidou, E., Udry, C., 1997. Estimation of type 3 Tobit models using symmetric trimming and pairwise comparisons. Journal of Econometrics 76, 107-128.

Honoré, B.E., Powell, J.L., 1994. Pairwise difference estimators of censored and truncated regression models. Journal of Econometrics 64, 241-278.

Horowitz, J.L., 1986. A distribution-free least squares estimator for censored linear regression models. Journal of Econometrics 32, 59-84.

Horowitz, J.L., 1988. Semiparametric M-estimation of censored linear regression models. Advances in Econometrics 7, 45-83.

Horowitz, J.L., 1993. Semi-parametric and non-parametric estimation of quantal response models. In: Maddala, G.S., Rao, C.R., Vinod, H.D. (Eds.), Handbook of Statistics, Vol. 11. Elsevier, Amsterdam.

Jarque, C., 1987. An application of LDV models to household expenditure analysis in Mexico. Journal of Econometrics 36, 31-54.

Judge, G.C., Hill, R.C., Griffiths, W.E., Lütkepohl, H., Lee, T.-C., 1982. Introduction to the Theory and Practice of Econometrics. Wiley, New York.

Khan, S., Powell, J.L., 1999. Two-step quantile estimation of the censored regression model. Manuscript, University of Rochester.

Koenker, R., Bassett Jr., G.S., 1978. Regression quantiles. Econometrica 46, 33-50.

Koenker, R., Bassett Jr., G.S., 1982. Robust tests for heteroskedasticity based on regression quantiles. Econometrica 50, 43-61.

Lewbel, A., Linton, O.B., 1998. Nonparametric censored regression. Manuscript, unpublished.

Maddala, G.S., 1995. Specification tests in limited dependent variable models. In: Maddala, G.S., Phillips, P.C.B., Srinivasan, T.N. (Eds.), Advances in Econometrics and Quantitative Economics. Oxford, Blackwell, pp. 1-49.

Melenberg, B., Van Soest, A., 1996. Parametric and semiparametric modelling of vacation expenditures. Journal of Applied Econometrics 11, 59-76.

Moon, C.-G., 1989. A Monte Carlo comparison of semiparametric Tobit estimators. Journal of Applied Econometrics 4, 361-382.

Nawata, K., 1990. Robust estimation based on group-adjusted data in censored regression models. Journal of Econometrics 43, 337-362.

Newey, W.K., McFadden, D., 1994. Estimation and hypothesis testing in large samples. In: Engle, R.F., McFadden, D. (Eds.), Handbook of Econometrics, Vol. 4. North-Holland, Amsterdam.

Newey, W.K., Stoker, T.M., 1993. Efficiency of weighted average derivative estimators and index models. Econometrica 61, 1199-1223.

Powell, J.L., 1984. Least absolute deviations estimation for the censored regression model. Journal of Econometrics 25, 303-325.

Powell, J.L., 1986a. Censored regression quantiles. Journal of Econometrics 32, 143-155.

Powell, J.L., 1986b. Symmetrically trimmed least squares estimation of Tobit models. Econometrica $54,1435-1460$.

Powell, J.L., Stock, J.H., Stoker, T.M., 1989. Semiparametric estimation of index coefficients. Econometrica 57, 1404-1430.

Sherman, R.P., 1994. U-processes in the analysis of a generalized semiparametric regression estimator. Econometric Theory 10, 372-395.

Tobin, J., 1958. Estimation of relationships for limited dependent variables. Econometrica 26, 24-36.

White, H., 1980. A heteroskedasticity-consistent covariance matrix estimator and a direct test for heteroskedasticity. Econometrica 48, 817-838. 